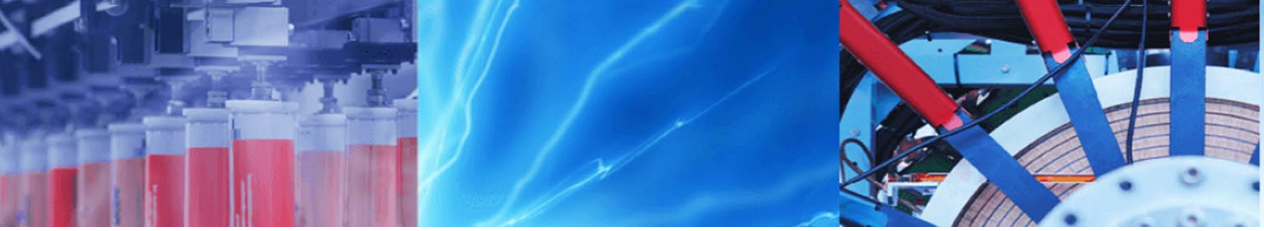

Research Article

\title{
Bioavailability of arsenic in agricultural soils under the influence of different soil properties
}

\author{
Suman B. Singh ${ }^{1,2} \cdot$ Pankaj Kumar Srivastava ${ }^{1}$
}

Received: 29 October 2019 / Accepted: 21 December 2019 / Published online: 3 January 2020

(c) Springer Nature Switzerland AG 2020

\begin{abstract}
The major arsenic contamination in soil is resulting from the application of contaminated irrigation groundwater, which may further enter into the food chain via dietary intake. The severity of arsenic contamination in soil is regulated by the total arsenic content and its bioavailability. The bioavailable fraction of arsenic in soil is measured as the most considerable fraction, while assessing the latent risk arising from it. Various soil properties and their interactions with soil arsenic would give a better understanding of its availability in soil. The study was carried out to look into different bioavailable fractions of arsenic in soil and their relationships with different soil properties in the arsenic-prone areas of West Bengal, India. The results showed that the bioavailable soil arsenic fractions were in the order of water soluble $<$ weakly adsorbed $<$ specifically adsorbed. The arsenic content in paddy soils was significantly $(p<0.05)$ influenced by bulk density, clay, $\mathrm{pH}$, organic matter, available calcium, available phosphorus, and available sulfur contents in soil. Soil enzyme activities of dehydrogenase, hydrolase, and glucosidase were also positively regulating the soil arsenic content. The study provides a better understanding of the interaction between different soil properties and soil arsenic content for developing the site-specific soil arsenic remediation strategies.
\end{abstract}

Keywords Contamination · Bioavailable fractions · Soil properties · Groundwater · Paddy · Arsenic

\section{Introduction}

Contamination of soils by arsenic is a major environmental concern. Arsenic (As) is considered as a toxic metalloid, a class-I carcinogen which is naturally distributed in water and soils in different parts of the world mostly in Southeast Asia [20,32]. Soil contamination with As originates both geogenically and anthropogenically. Arsenic contamination of groundwater has been recognized in 105 countries with an estimate of 200 million humans exposed worldwide to arsenic toxicity [24, 25].

Arsenic contamination is widespread in many countries, such as Argentina, Bangladesh, Cambodia, Chile, Taiwan, China, Thailand, the USA, and especially Bangladesh and West Bengal, India $[1,3,7,8]$. The arsenic pollution occurs in the Ganga-Meghna-Brahmaputra (GMB) plains of India, and Bangladesh is the worst affected area among the world arsenic scenario. In GMB plain alone, currently, more than 100 million people are potentially at risk from groundwater arsenic contamination [12]. Exposure from consumption of arsenic-contaminated drinking water and food crops has resulted in adverse health impacts on humans, such as stomach pain, circulatory problems, keratosis, cancer, and skin lesions. Arsenic contamination of groundwater in West Bengal was first reported in the early 1980s. The permissible limits of arsenic in drinking water $\left(10 \mu \mathrm{g} \mathrm{I}^{-1}\right)$ and agricultural soil $\left(20 \mathrm{mg} \mathrm{kg}^{-1}\right)$ have been given by the Agency for Toxic Substances and Disease Registry (ASTDR), 1985 [15, 43]. Arsenic contamination in soil and water is a serious threat due to the transferring of

Pankaj Kumar Srivastava, drpankajk@gmail.com | 'Division of Environmental Technologies, CSIR-National Botanical Research Institute, Rana Pratap Marg, Lucknow 226001, India. ${ }^{2}$ Academy of Scientific and Innovative Research (AcSIR), Ghaziabad 201002, India. 
arsenic through irrigation water to the soil-plant system. The arsenic contamination in soil is regulated by its content and availability in soil. The bioavailable fractions of arsenic are actually responsible for the toxicity compared to the total content. The bioavailable fraction is directly interacting with the living organism in soils. It is considered the most important fraction to assess the latent ecotoxicological threats. There is also a need to understand the important factors, which affect As availability, mobility, and chemistry in soils. These important factors are soil properties, solid composition, As-bearing phases, adsorption-desorption processes, biological transformations, and the volatilization of As in soil $[32,16]$. Moreover, organic compounds and oxide/hydroxides of $\mathrm{Fe}, \mathrm{Zn}, \mathrm{Al}$, and $\mathrm{Mn}$ also influence the amount of bioavailable arsenic in soil $[10,32]$ by forming various compounds with different binding forces in the soil-water system. The total arsenic content in soil may be a good indicator to measure its degree of contamination, but insufficient for assessment of its environmental impact without considering the arsenic bioavailable fractions. Different fractions of arsenic in soil can provide useful information for the assessment of arsenic bioavailability and toxicity in soils [23]. In order to devise remediation strategies, it is required to understand the relationship between arsenic (As) bioavailable fractions in soil and other soil properties, which may regulate the arsenic mobility in the contaminated soils. The objective of the study was to estimate bioavailable fractions of arsenic in soil in the arsenic-contaminated region. The interaction between the bioavailability of arsenic in soil and different soil properties was also assessed to find out the major soil parameters regulating the arsenic bioavailability in soil.

\section{Materials and methods}

\subsection{Study site}

Soil samples were collected from nine administrative blocks of five districts in the state of West Bengal, India. These five districts have been reported as arsenic-contaminated zone in the Lower Ganga plains, Bengal Basin of the Indo-Gangetic alluvium in South Asia [9]. The five districts were Hooghly $\left(22^{\circ} 53^{\prime} 45^{\prime \prime} \mathrm{N}\right.$ and $\left.88^{\circ} 22^{\prime} 07^{\prime \prime} \mathrm{E}\right)$, Nadia $\left(23^{\circ} 47^{\prime} 10^{\prime \prime} \mathrm{N}, 88^{\circ} 55^{\prime} 65^{\prime \prime} \mathrm{E}\right)$, Murshidabad $\left(24^{\circ} 22^{\prime} 90^{\prime \prime} \mathrm{N}\right.$, $\left.88^{\circ} 24^{\prime} 61^{\prime \prime} \mathrm{E}\right)$, Burdwan $\left(23^{\circ} 23^{\prime} 24^{\prime \prime} \mathrm{N}, 8^{\circ} 86^{\prime} 15^{\prime \prime} \mathrm{E}\right)$, and

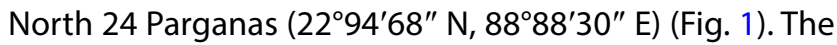
climate of the study area is tropical to humid subtropical. The arsenic contamination in the study area is of geogenic origin and occurred through exploitation of groundwater for irrigation. None of any anthropogenic pollution sources were spotted in the study area.

\subsection{Sampling}

Composite soil samples from agricultural fields were collected in two phases from arsenic-contaminated areas of five districts of West Bengal during May-July 2015 and July 2016. The soil was vertisols and clay-loam type. The field of soil sampling was being used for cultivating paddy in the region. Soil sampling was made to a soil depth of $5-15 \mathrm{~cm}$ from the surface. Thirty soil samples were collected in the Phase-I from ten different distantly located villages/sites of these five districts in West Bengal. Fifteen soil samples were further collected in the Phase-Il from the 15 different distant sites (agriculture fields) at three villages, in which arsenic contents were found higher during the Phase-I. The Phase-II was conducted to augment the number of representative samples of these arsenic-contaminated sites of three villages of Phase-I. Samples were stored at low temperature for transportation to the laboratory and stored at $4{ }^{\circ} \mathrm{C}$ until further analysis. The samples were air-dried, crushed, and passed through a 2-mm sieve for determining all soil parameters, and moist samples were stored at $4{ }^{\circ} \mathrm{C}$ for soil enzyme analysis.

In the Phase-II, 15 groundwater samples $(n=3)$ were also collected from irrigation water sources in July 2016 from three villages. The water samples were collected in the sterilized polyethylene bottles fitted with a liquidtight stopper after running the tube well for $10 \mathrm{~min}$. The water samples were then immediately acidified with $1 \%$ $\mathrm{HNO}_{3}$ and analyzed for arsenic content within 7 days of sampling.

\subsection{Soil physicochemical analysis}

The physicochemical analysis of soil samples was carried out in triplicates. The $\mathrm{pH}$ and electrical conductivity (EC) of soils were measured by using the Orion meter in soil/ water a ratio of 1:2.5 (w/v). Soil water holding capacity (WHC) was measured gravimetrically according to Keen's box method by weighing water-saturated and ovendried samples $\left(24 \mathrm{~h}\right.$ at $105^{\circ} \mathrm{C}$ ) [6]. The total organic carbon (TOC) was estimated by the method of Walkley and Black (1934). Organic matter (OM) values were obtained from the estimated organic carbon $(\mathrm{OC})$ values using the following formula $\{O M=1.724$ (Van Bemelen factor) $\times O C$ [ [27]. The available nitrogen was determined by using the Kjeldahl method [6]. The microbial biomass carbon (MBC) was determined by the chloroform-fumigation-extraction Vance method [37-39]. The available sodium ( $\mathrm{Na}$ ), potassium (K), and calcium ( $\mathrm{Ca}$ ) contents were measured by the extraction method by using flame 
Fig. 1 Study sites in the state of West Bengal, India

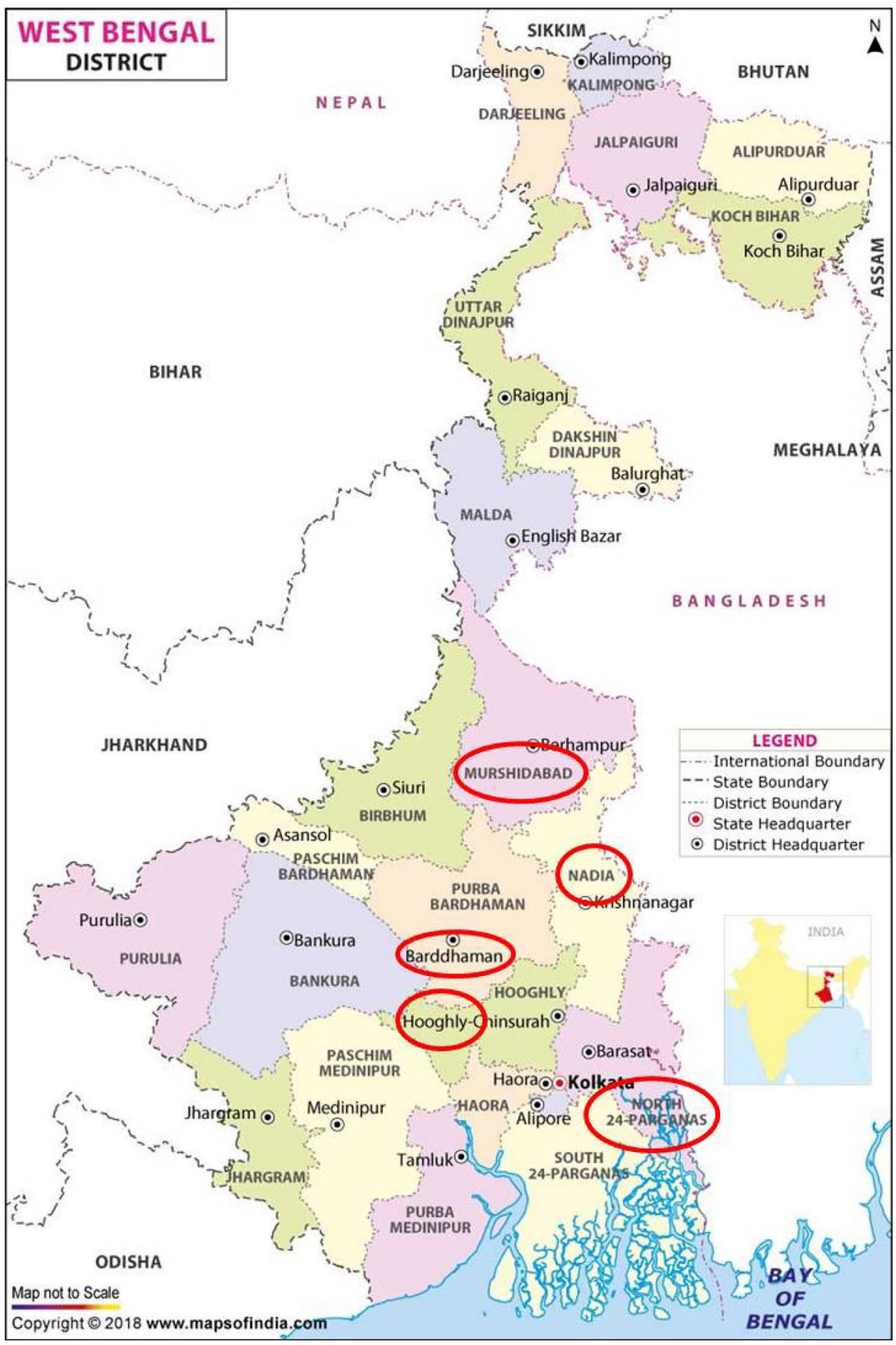

photometer (Systronix-128) (Jackson 1962). Available phosphorus $(P)$ content was determined after the extraction of sample in $2 \%$ sodium bicarbonate solution [26].

Moisture content was estimated using the method of Bhattacharya et al. [4]. The bulk density (gravity bottles) was estimated according to the method as earlier described by Srivastava et al. [37-39]. The soil texture was estimated by the Bouyoucous hydrometer method.
The soil available sulfur-sulfate was determined by the method of Tabatabai and Bremner [40]. Soil enzyme activities were done in moist soil samples for determining dehydrogenase (DHA), fluorescein diacetate hydrolase (FDA), arylsulfatase (ASA), alkaline phosphatase (APA) $\beta$-glucosidase (BG), cellulase (CeA), and protease (PA) using the standard methods of Dick et al. [13] and Srivastava et al. [37]. 


\subsection{Total arsenic content}

The digestion of air-dried soil samples $(0.25 \mathrm{~g})$ was made by using $\mathrm{HNO}_{3}: \mathrm{H}_{2} \mathrm{O}_{2}(5: 1)$. Cooled digested soil samples and acidified water samples $(5 \mathrm{ml})$ were filtered through Whatman syringe filter $0.2 \mu \mathrm{m}$, followed by a volume make up to $30 \mathrm{ml}$ with deionized water. Total As content in water and soil samples was estimated by using inductively coupled plasma mass spectrometry (ICP-MS, Agilent 7500 ce).

\subsection{Soil bioavailable arsenic fractions}

The fractionation of As in soil samples was performed using the sequential extraction procedure described by Wenzel et al. [42]. The bioavailable arsenic fractions were determined as water-soluble, weakly adsorbed extractable in $0.5 \mathrm{M} \mathrm{NaHCO}_{3}$ and specifically adsorbed extractable in $0.05 \mathrm{M} \mathrm{NH}_{4} \mathrm{H}_{2} \mathrm{PO}_{4}$. The aliquots were taken and centrifuged at $3000 \mathrm{rpm}$ for $15 \mathrm{~min}$ at room temperature, filtered through Whatman filter paper no. 40. Extracted bioavailable arsenic fractions in soil samples were estimated using ICP-MS.

\subsection{Quality control and quality assurance}

Quality control measures for each batch including calibration with reference samples, blanks, and replicate analysis were followed throughout the analysis in order to ensure reliable analytical data. Sample digestion batches were accompanied by analytical blanks, rice flour certified reference material (CRM) (NIST 1568a) and blank spikes. The accuracy of the sequential extraction was also evaluated by analyzing the same CRM. Mean CRM and spike recoveries of total As were $90 \%( \pm 3.1 ; n=5)$ and $85 \%( \pm 2.8$; $n=5$ ), respectively. The method detection limit for As was $1 \mu \mathrm{g} \mathrm{I} \mathrm{I}^{-1}$.

\subsection{Statistical analysis}

The data were subjected to statistical analysis using analysis of variance (ANOVA) - the general linear model (GLM) to study significant differences at $p<0.05$ among the values of different parameters by using SPSS 10.0. Comparison of means and levels of significance were evaluated according to Duncan's multiple range test (DMRT).

Multivariate principal component analysis (PCA) was used to reduce a large number of variables to representative factors called "Principal Components." Principal components were computed by factors that are the eigenvectors of the correlation matrix of the original data. PCA as the multivariate analytical tool was used to reduce a set of original variables and to extract a small number of latent factors (principal components, PCs) for analyzing relationships among the observed variables. The number of factors extracted from the variables was determined by a screen test according to Kaiser's rule. This criterion retained only factors with eigenvalues that exceed one. Varimax-rotated factor loadings greater than 0.50 were underlined to assist the interpretation of the meanings of the factors. In order to find possible tendencies in the samples and the discriminated power of the variables, PCA was applied. The components accounting for the maximum variance in the PCA output were chosen as significantly delineating the relationships between total soil arsenic content with bioavailable fractions of arsenic and different soil properties.

\section{Results}

In the study, 10 and 15 sites were surveyed during Phase-I and Phase-II, respectively. Physicochemical characteristics of paddy soil samples in ten villages were found significantly different (Tables 1 and 2). The $\mathrm{pH}$ values of all the soil samples were alkaline in nature. The $\mathrm{EC}$ values of soils ranged between 0.145 and $0.344 \mathrm{dS} \mathrm{m}^{-1}$ (Table 2). The soil was clay to clay loam. Clay contents ranged from 28 to $62 \%$ in all the sites covered in Phase-I and found correlated with arsenic content in soils $\left(r^{2}=0.96 ; p<0.05\right)$. The water holding capacity (WHC) ranged from 53 to $61 \%$. The bulk density (BD) ranged between 1.27 and $1.46 \mathrm{gm} \mathrm{cm}^{-3}$ with a mean value of $1.32 \mathrm{gm} \mathrm{cm}^{-3}$ (Table 1).

The contents of available phosphorus (36-58 $\mathrm{mg} \mathrm{kg}^{-1}$ ), available sulfur $\left(8.86-30.41 \mathrm{mg} \mathrm{kg}^{-1}\right)$, and available $\mathrm{K}\left(71.27-133 \mathrm{mg} \mathrm{kg}^{-1}\right)$ in soils of ten sites are given in Table 2 . The soils were having high available $\mathrm{Ca}\left(3303-7245 \mathrm{mg} \mathrm{kg}^{-1}\right)$ and available $\mathrm{Na}$ (56.35-227.44 $\mathrm{mg} \mathrm{kg}^{-1}$ ) contents (Table 2). The TOC, OM, and $\mathrm{MBC}$ were ranged normal to high as $2.32-5.64 \%$,

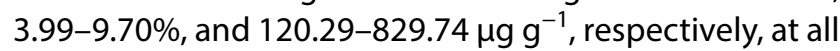
sites (Table 2).

Enzyme activities for PA, APA, and ASA found low in soil containing higher arsenic content, except DHA, FDA, BGA, and CeA (Table 3). The mean values for DHA, FDA, APA, and ASA ranged from 12.66-21.68, 13.30-20.20, 19.42-118, and $47.51-191.44 \mu^{-1} \mathrm{~g} \mathrm{~g}^{-1}$ soil $^{-1}$, respectively. Soil arsenic content was significantly $(p<0.05)$ and negatively correlated with the enzyme activities. Highest values for $\mathrm{DHA}$, FDA, BGA, and CeA in soil samples were recorded in Pipligram. The result showed DHA and FDA values were highest in highly contaminated sites.

In soil, the total arsenic content ranged from 6.64 to $31.11 \mathrm{mg} \mathrm{kg}^{-1}$ (Table 4). The arsenic contents in soils of the studied area were found above (32-520\%) the general naturally occurring soil arsenic content, i.e., $5 \mathrm{mg} \mathrm{kg}^{-1}$ [28]. However, arsenic levels in some of the villages were 
Table 1 Soil physical properties of ten villages in West Bengal

\begin{tabular}{lcccc}
\hline Ten villages in the Phase-I & $\mathrm{BD}\left(\mathrm{gm} \mathrm{cm}^{-3}\right)$ & WHC (\%) & Moisture (\%) & Texture \\
\hline $\begin{array}{l}\text { Chinsurah } \\
\text { (Hooghly) }\end{array}$ & $1.36^{\mathrm{cd}} \pm 0.03$ & $58.52^{\mathrm{de}} \pm 0.47$ & $51.04^{\mathrm{e}} \pm 0.25$ & Clay loam \\
$\begin{array}{l}\text { Santipur } \\
\text { (Nadia) }\end{array}$ & $1.27^{\mathrm{b}} \pm 0.03$ & $57.43^{\mathrm{d}} \pm 0.47$ & $24.51^{\mathrm{a}} \pm 0.16$ & Clay \\
$\begin{array}{l}\text { Dhomkal } \\
\text { (Murshidabad) }\end{array}$ & $1.46^{\mathrm{d}} \pm 0.06$ & $53.05^{\mathrm{a}} \pm 0.18$ & $26.49^{\mathrm{b}} \pm 0.79$ & Sandy clay \\
$\begin{array}{l}\text { Beldanga-I } \\
\text { (Murshidabad) }\end{array}$ & $1.35^{\mathrm{c}} \pm 0.05$ & $55.00^{\mathrm{b}} \pm 0.54$ & $24.05^{\mathrm{a}} \pm 0.29$ & Clay loam \\
$\begin{array}{l}\text { Purbasthali-I } \\
\text { (Burdwan) }\end{array}$ & $1.42^{\mathrm{d}} \pm 0.04$ & $56.09^{\mathrm{c}} \pm 0.27$ & $26.07^{\mathrm{a}} \pm 0.06$ & Silty clay loam \\
$\begin{array}{l}\text { Ranaghat-I } \\
\text { (Nadia) }\end{array}$ & $1.28^{\mathrm{b}} \pm 0.04$ & $59.15^{\mathrm{e}} \pm 0.32$ & $24.94^{\mathrm{a}} \pm 0.01$ & Clay \\
$\begin{array}{l}\text { Chakdah } \\
\text { (Nadia) }\end{array}$ & $1.15^{\mathrm{a}} \pm 0.12$ & $59.80^{\mathrm{e}} \pm 0.33$ & $24.01^{\mathrm{a}} \pm 0.46$ & Clay \\
$\begin{array}{l}\text { Ghentughachi } \\
\text { (Nadia) }\end{array}$ & $1.39^{\mathrm{cd}} \pm 0.07$ & $59.01^{\mathrm{e}} \pm 0.03$ & $27.96^{\mathrm{b}} \pm 0.30$ & Clay loam \\
$\begin{array}{l}\text { Pipligram-Gaighata } \\
\text { (N 24 Parganas) }\end{array}$ & $1.30^{\mathrm{bc}} \pm 0.03$ & $57.85^{\mathrm{d}} \pm 0.28$ & $32.78^{\mathrm{c}} \pm 0.32$ & Clay \\
$\begin{array}{l}\text { Haringhata } \\
\text { (Nadia) }\end{array}$ & $1.29^{\mathrm{b}} \pm 0.01$ & $61.80^{\mathrm{f}} \pm 0.43$ & $38.09^{\mathrm{d}} \pm 0.91$ & Clay \\
\hline
\end{tabular}

$\mathrm{BD}$, bulk density; WHC, water holding capacity

The significant differences among the Mean \pm SE $(n=3)$ values of a column are mentioned by different alphabets (in superscript) $(p<0.05)$ found above the threshold limit of $20 \mathrm{mg} \mathrm{kg}^{-1}$ given for agricultural soils [15]. Table 4 shows the total arsenic contents and its sequential extracted bioavailable fractions in the soil samples. The results showed significant differences $(p<0.05)$ among all the bioavailable fractions at different sites. On average, the water-soluble fractions accounted for 3-5\% of total As, whereas weakly adsorbed fraction accounted for 5-8\%. The specifically sorbed fraction comprised $7-12 \%$ of total As (Table 4). Remaining As accounted for $75-85 \%$ of the total As would be bounded or residual fractions (Table 4). The results showed arsenic partitioning following a descending order: residual fractions (or well-crystallized hydrous oxides of Fe and $\mathrm{Al}$, amorphous and poorly crystalline hydrous oxides of Fe and Al) $\geq$ specifically sorbed $>$ weakly sorbed $>$ water soluble. The soil arsenic content found higher at Ghentughachi, Gotera, and Pipligram during the Phase-I. The results of the Phase-Il for the arsenic contents in groundwater and soils of all 15 sites of these three villages are presented in Tables 5, 6, 7 and 8.

In Phase-II, the total arsenic content ranged from 15.03 to $32.10 \mathrm{mg} \mathrm{kg}^{-1}$ and 112.3 to $558.1 \mathrm{\mu g} \mathrm{I}^{-1}$ in soil and groundwater, respectively, among different sites of Ghentughachi, Gotera and Pipligram (Table 5). Among all the 15 different sites of Phase-II, bioavailable fractions were significantly varied $(p<0.05)$ (Table 5$)$. The results showed that the water-soluble fractions ranged between 0.467 and $1.380 \mathrm{mg} \mathrm{kg}^{-1}$ of total As, weakly adsorbed fraction between 0.85 and $2.65 \mathrm{mg} \mathrm{kg}^{-1}$, and specifically sorbed fraction between 1.308 and $3.360 \mathrm{mg} \mathrm{kg}^{-1}$. The chemical properties of soils obtained in the Phase-Il are given in Tables 6 and 7. The enzyme activities were lower in higher arsenic-containing soils (Table 8). The relationships between total soil arsenic content with bioavailable fractions of arsenic and different soil properties were studied using PCA.

The PCA biplot of soil arsenic content of different sites of the Phase-I showed $98.74 \%$ of the total variance (Fig. 2). The PCA biplot of soil physical properties and soil arsenic content resulted in $83.39 \%$ of total variance (Fig. 3). Soil arsenic content was negatively correlated with the soil bulk density and positively correlated with soil moisture and water holding capacity at all the sites. The PCA biplot between soil arsenic and soil chemical properties resulted in $77.03 \%$ of the total variance, depicting that soil TOC, $\mathrm{MBC}, \mathrm{OM}$, and $\mathrm{Ca}$ contents were positively correlated with soil arsenic content (Fig. 4) at all the sites. Figure 5 explains PCA biplot performed between soil enzyme activities and soil arsenic content accounted for $76.14 \%$ of the total variance, showing that DHA, FDA, CeA, and BGA soil enzyme activities were positively correlated with the soil arsenic contents, whereas the AP, PA, and ASA activities were negatively correlated at all the sites. The PCA biplot of total As content in the groundwater and soil samples of Phase-II resulted in $85.73 \%$ of total variance (Fig. 6). Figures 7, 8, and 9 show similar trend of the results between soil arsenic 


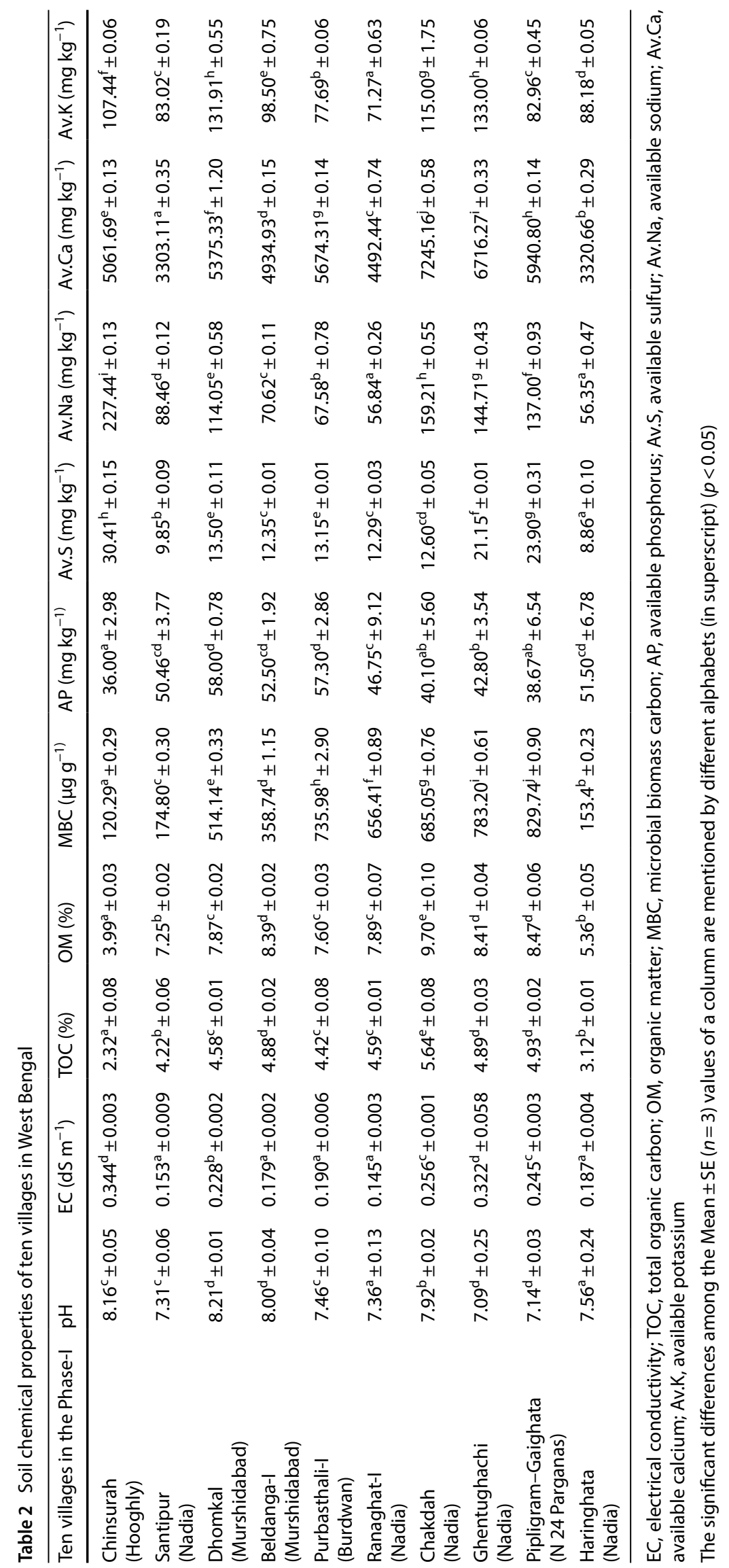


Table 3 Enzyme activities of soil of ten villages in West Bengal

\begin{tabular}{|c|c|c|c|c|c|c|c|}
\hline Ten villages in the Phase-I & DHA & FDA & APA & BG & PA & $\mathrm{CeA}$ & ASA \\
\hline $\begin{array}{l}\text { Chinsurah } \\
\text { (Hooghly) }\end{array}$ & $12.66^{\mathrm{a}} \pm 0.46$ & $13.30^{\mathrm{a}} \pm 0.15$ & $79.96^{\mathrm{e}} \pm 1.34$ & $18.70^{\mathrm{a}} \pm 0.12$ & $763.77^{9} \pm 1.37$ & $0.749^{\mathrm{a}} \pm 0.016$ & $191.44^{i} \pm 0.17$ \\
\hline $\begin{array}{l}\text { Santipur } \\
\text { (Nadia) }\end{array}$ & $16.88^{\mathrm{C}} \pm 0.04$ & $17.60^{d} \pm 0.17$ & $94.02^{\mathrm{g}} \pm 0.04$ & $27.47^{d} \pm 0.02$ & $221.89^{c} \pm 0.03$ & $1.389^{\mathrm{a}} \pm 0.151$ & $155.77^{9} \pm 0.26$ \\
\hline $\begin{array}{l}\text { Dhomkal } \\
\text { (Murshidabad) }\end{array}$ & $14.91^{b} \pm 0.01$ & $15.16^{\mathrm{C}} \pm 0.05$ & $80.02^{\mathrm{e}} \pm 0.06$ & $18.67^{\mathrm{a}} \pm 0.01$ & $612.70^{f} \pm 2.71$ & $1.935^{b} \pm 0.014$ & $158.58^{h} \pm 0.46$ \\
\hline $\begin{array}{l}\text { Beldanga-I } \\
\text { (Murshidabad) }\end{array}$ & $13.58^{\mathrm{a}} \pm 0.02$ & $14.25^{\mathrm{b}} \pm 0.01$ & $24.64^{c} \pm 0.29$ & $23.78^{\complement} \pm 0.02$ & $402.61^{f} \pm 0.39$ & $3.982^{\mathrm{c}} \pm 0.317$ & $150.94^{f} \pm 0.43$ \\
\hline $\begin{array}{l}\text { Purbasthali-I } \\
\text { (Burdwan) }\end{array}$ & $21.68^{d} \pm 0.09$ & $20.20^{9} \pm 0.08$ & $58.59^{d} \pm 0.35$ & $23.52^{b} \pm 0.03$ & $228.52^{d} \pm 0.20$ & $7.027^{\mathrm{e}} \pm 0.030$ & $47.51^{a} \pm 0.16$ \\
\hline $\begin{array}{l}\text { Ranaghat-I } \\
\text { (Nadia) }\end{array}$ & $15.21^{b} \pm 0.02$ & $16.96^{d} \pm 0.08$ & $84.67^{f} \pm 0.22$ & $39.42^{9} \pm 0.01$ & $175.30^{b} \pm 0.32$ & $8.940^{f} \pm 0.301$ & $52.63^{c} \pm 0.15$ \\
\hline $\begin{array}{l}\text { Chakdah } \\
\text { (Nadia) }\end{array}$ & $18.16^{d} \pm 0.01$ & $18.57^{\mathrm{e}} \pm 0.02$ & $19.42^{\mathrm{a}} \pm 0.49$ & $31.65^{e} \pm 0.02$ & $107.45^{\mathrm{a}} \pm 0.17$ & $10.81^{g} \pm 0.376$ & $47.91^{a} \pm 0.02$ \\
\hline $\begin{array}{l}\text { Ghetugachi } \\
\text { (Nadia) }\end{array}$ & $13.88^{a} \pm 0.02$ & $14.28^{b} \pm 0.03$ & $119.8^{\mathrm{h}} \pm 0.27$ & $23.96^{c} \pm 0.03$ & $229.63^{d} \pm 0.36$ & $7.960^{\mathrm{e}} \pm 0.065$ & $60.67^{d} \pm 0.15$ \\
\hline $\begin{array}{l}\text { Pipligram-Gaighata } \\
\text { (N } 24 \text { Parganas) }\end{array}$ & $20.26^{d} \pm 0.03$ & $19.41^{f} \pm 0.01$ & $22.04^{b} \pm 0.08$ & $33.77^{f} \pm 0.04$ & $176.76^{b} \pm 0.08$ & $11.84^{\mathrm{h}} \pm 0.058$ & $66.37^{e} \pm 0.17$ \\
\hline $\begin{array}{l}\text { Haringhata } \\
\text { (Nadia) }\end{array}$ & $16.11^{\mathrm{c}} \pm 0.04$ & $17.21^{d} \pm 0.02$ & $118.0^{\mathrm{h}} \pm 0.07$ & $18.48^{\mathrm{a}} \pm 0.02$ & $395.45^{\mathrm{e}} \pm 1.70$ & $5.850^{d} \pm 0.374$ & $50.69^{b} \pm 0.09$ \\
\hline
\end{tabular}

DHA (dehydrogenase activity) as $\mu \mathrm{g} \mathrm{TPF} \mathrm{g} \mathrm{soil}{ }^{-1} 24 \mathrm{~h}^{-1}$; FDA (fluorescein diacetate) as $\mu \mathrm{g}$ fluorescein $\mathrm{g} \mathrm{soil}{ }^{-1} 2 \mathrm{~h}^{-1}$; APA (alkaline phosphatase activity) as $\mu \mathrm{g} \rho$-NP g soil ${ }^{-1} \mathrm{~h}^{-1}$; BG ( $\beta$-glucosidase) as $\mu \mathrm{g} \rho$-NP g soil ${ }^{-1} \mathrm{~h}^{-1}$; PA (protease activity) as $\mu \mathrm{g} \mathrm{Tyr} \mathrm{g} \mathrm{soil}{ }^{-1} 2 \mathrm{~h}^{-1}$; CeA (cellulase activity) as $\mu$ g glucose $g$ soil $^{-1} 24 \mathrm{~h}^{-1}$; ASA (arylsulfatase) as $\mu \mathrm{g} \rho$-NP g soil ${ }^{-1} \mathrm{~h}^{-1}$

The significant differences among the Mean \pm SE $(n=3)$ values of a column are mentioned by different alphabets (in superscript) $(p<0.05)$

Table 4 Total and bioavailable fractions of soil arsenic $\left(\mathrm{mg} \mathrm{kg}^{-1}\right)$ of ten villages in West Bengal

\begin{tabular}{|c|c|c|c|c|c|}
\hline \multirow[t]{2}{*}{ Ten villages in the Phase-I } & \multirow[t]{2}{*}{ TAs } & \multicolumn{4}{|c|}{ Bioavailable arsenic } \\
\hline & & $\mathrm{BA} / \mathrm{WS}$ & BA/WA & $\mathrm{BA} / \mathrm{SA}$ & $\mathrm{BA} / \mathrm{RA}$ \\
\hline $\begin{array}{l}\text { Chinsurah } \\
\text { (Hooghly) }\end{array}$ & $6.64^{a} \pm 0.02$ & $0.332^{\mathrm{a}} \pm 0.057$ & $0.464^{a} \pm 0.003$ & $0.531^{\mathrm{ab}} \pm 0.001$ & $5.312^{\mathrm{a}} \pm 0.004$ \\
\hline $\begin{array}{l}\text { Santipur } \\
\text { (Nadia) }\end{array}$ & $8.95^{b} \pm 0.03$ & $0.358^{a} \pm 0.039$ & $0.447^{\mathrm{a}} \pm 0.002$ & $0.716^{\mathrm{abc}} \pm 0.002$ & $7.428^{b} \pm 0.020$ \\
\hline $\begin{array}{l}\text { Dhomkal } \\
\text { (Murshidabad) }\end{array}$ & $11.94^{c} \pm 0.07$ & $0.358^{a} \pm 0.005$ & $0.835^{\mathrm{e}} \pm 0.001$ & $0.955^{\mathrm{ab}} \pm 0.282$ & $9.79^{d} \pm 0.061$ \\
\hline $\begin{array}{l}\text { Beldanga-I } \\
\text { (Murshidabad) }\end{array}$ & $9.71^{b} \pm 0.08$ & $0.291^{\mathrm{a}} \pm 0.088$ & $0.485^{\mathrm{a}} \pm 0.008$ & $0.679^{\mathrm{ab}} \pm 0.026$ & $8.253^{C} \pm 0.029$ \\
\hline $\begin{array}{l}\text { Purbasthali-I } \\
\text { (Burdwan) }\end{array}$ & $12.5^{c} \pm 0.26$ & $0.375^{\mathrm{a}} \pm 0.147$ & $0.750^{d} \pm 0.024$ & $1.125^{\mathrm{cd}} \pm 0.028$ & $10.25^{d} \pm 0.156$ \\
\hline $\begin{array}{l}\text { Ranaghat-I } \\
\text { (Nadia) }\end{array}$ & $17.7^{d} \pm 0.06$ & $0.531^{\mathrm{a}} \pm 0.005$ & $0.885^{\mathrm{e}} \pm 0.001$ & $1.239^{d} \pm 0.010$ & $15.04^{\mathrm{e}} \pm 0.358$ \\
\hline $\begin{array}{l}\text { Chakdah } \\
\text { (Nadia) }\end{array}$ & $31.11^{f} \pm 0.31$ & $1.55^{d} \pm 0.037$ & $2.170^{g} \pm 0.067$ & $4.040^{f} \pm 0.009$ & $23.33^{g} \pm 0.009$ \\
\hline $\begin{array}{l}\text { Ghetugachi } \\
\text { (Nadia) }\end{array}$ & $24.7^{e} \pm 0.08$ & $1.235^{c} \pm 0.021$ & $1.729^{f} \pm 0.012$ & $2.470^{\mathrm{e}} \pm 0.020$ & $21.73^{f} \pm 0.026$ \\
\hline $\begin{array}{l}\text { Pipligram-Gaighata } \\
\text { (N } 24 \text { Parganas) }\end{array}$ & $24.75^{\mathrm{e}} \pm 0.27$ & $0.991^{b} \pm 0.008$ & $1.980^{f} \pm 0.114$ & $2.970^{\mathrm{e}} \pm 0.342$ & $21.28^{f} \pm 0.008$ \\
\hline $\begin{array}{l}\text { Haringhata } \\
\text { (Nadia) }\end{array}$ & $9.75^{\mathrm{b}} \pm 0.41$ & $0.390^{a} \pm 0.021$ & $0.585^{\mathrm{b}} \pm 0.022$ & $0.877^{b c d} \pm 0.010$ & $7.89^{b c} \pm 0.005$ \\
\hline
\end{tabular}

TAs, total arsenic; BA/WS, bioavailable arsenic water soluble; BA/WA, bioavailable arsenic weakly adsorbed; BA/SA, bioavailable arsenic specifically adsorbed; BA/RA, bioavailable arsenic residual

The significant differences among the Mean \pm SE $(n=3)$ values of a column are mentioned by different alphabets (in superscript) $(p<0.05)$ 
Table 5 Arsenic content in soil and groundwater (GW) in 15 different sites of three villages in West Bengal

\begin{tabular}{|c|c|c|c|c|c|c|}
\hline \multirow[t]{2}{*}{15 sites of three villages in the Phase-II } & \multirow{2}{*}{$\begin{array}{l}\text { Total soil arse- } \\
\text { nic }\left(\mathrm{mg} \mathrm{kg}^{-1}\right)\end{array}$} & \multicolumn{4}{|c|}{ Bioavailable arsenic in soil $\left(\mathrm{mg} \mathrm{kg}^{-1}\right)$} & \multirow{2}{*}{$\begin{array}{l}\text { Arsenic conten } \\
\text { in } \mathrm{GW}^{5} \mu \mathrm{g} \mathrm{I}^{-1}\end{array}$} \\
\hline & & BA/WS & BA/WA & $\mathrm{BA} / \mathrm{SA}$ & $\mathrm{BA} / \mathrm{RA}$ & \\
\hline $\begin{array}{l}\text { Ghetugachi, Chakdah, } \\
\text { Nadia (SusBis) }\end{array}$ & $26.23^{f} \pm 0.25$ & $1.310^{9} \pm 0.012$ & $1.830^{\mathrm{fg}} \pm 0.025$ & $2.623^{\mathrm{fg}} \pm 0.017$ & $20.450^{b} \pm 0.165$ & $356.4^{j} \pm 0.20$ \\
\hline $\begin{array}{l}\text { Ghetugachi, Chakdah, } \\
\text { Nadia (DipGho) }\end{array}$ & $29.49^{h} \pm 0.29$ & $0.884^{d} \pm 0.004$ & $1.761^{f} \pm 0.058$ & $2.940^{9} \pm 0.010$ & $23.880^{C} \pm 0.089$ & $428.1^{\mathrm{m}} \pm 0.03$ \\
\hline $\begin{array}{l}\text { Ghetugachi, Chakdah, } \\
\text { Nadia (HarCha) }\end{array}$ & $27.86^{9} \pm 0.52$ & $1.114^{f} \pm 0.002$ & $1.393^{d} \pm 0.003$ & $2.228^{\mathrm{de}} \pm 0.024$ & $23.120^{C} \pm 0.015$ & $402.4^{\prime} \pm 1.61$ \\
\hline $\begin{array}{l}\text { Ghetugachi, Chakdah, } \\
\text { Nadia (BikSar) }\end{array}$ & $25.55^{f} \pm 0.26$ & $1.022^{\mathrm{e}} \pm 0.007$ & $1.277^{c} \pm 0.001$ & $2.810^{f g} \pm 0.015$ & $20.440^{\mathrm{b}} \pm 0.012$ & $296.3^{f} \pm 0.87$ \\
\hline $\begin{array}{l}\text { Ghetugachi, Chakdah, } \\
\text { Nadia (PraBis) }\end{array}$ & $28.00^{9} \pm 0.07$ & $1.401^{\mathrm{h}} \pm 0.009$ & $1.960^{g} \pm 0.044$ & $3.360^{h} \pm 0.079$ & $21.280^{b c} \pm 0.032$ & $558.1^{n} \pm 0.32$ \\
\hline $\begin{array}{l}\text { Gotera, } \\
\text { Nadia (SuvMon) }\end{array}$ & $32.10^{i} \pm 0.01$ & $0.963^{\mathrm{e}} \pm 0.014$ & $1.605^{\mathrm{e}} \pm 0.003$ & $2.247^{\mathrm{de}} \pm 0.002$ & $27.280^{d} \pm 0.904$ & $342.2^{i} \pm 1.64$ \\
\hline $\begin{array}{l}\text { Gotera, } \\
\text { Nadia (ChhMon) }\end{array}$ & $27.60^{9} \pm 0.15$ & $1.380^{h} \pm 0.003$ & $1.932^{9} \pm 0.001$ & $2.484^{e f} \pm 0.005$ & $21.801^{b} \pm 0.066$ & $387.1^{k} \pm 2.61$ \\
\hline $\begin{array}{l}\text { Gotera, } \\
\text { Nadia (AnsMon) }\end{array}$ & $17.00^{\text {cd }} \pm 0.01$ & $0.510^{a} \pm 0.015$ & $0.85^{\mathrm{a}} \pm 0.009$ & $1.701^{b c} \pm 0.033$ & $13.940^{\mathrm{abc}} \pm 0.929$ & $112.3^{\mathrm{a}} \pm 1.16$ \\
\hline $\begin{array}{l}\text { Gotera, } \\
\text { Nadia (PraSan) }\end{array}$ & $15.03^{\mathrm{a}} \pm 0.02$ & $0.601^{b} \pm 0.004$ & $1.202^{C} \pm 0.028$ & $1.653^{\mathrm{ab}} \pm 0.001$ & $11.573^{\mathrm{a}} \pm 0.102$ & $323.2^{h} \pm 0.52$ \\
\hline $\begin{array}{l}\text { Gotera, } \\
\text { Nadia (GanBar) }\end{array}$ & $17.03^{d} \pm 0.15$ & $0.851^{c d} \pm 0.011$ & $1.192^{C} \pm 0.006$ & $2.043^{\text {cd }} \pm 0.019$ & $12.942^{\mathrm{a}} \pm 0.572$ & $362.4^{\mathrm{h}} \pm 1.31$ \\
\hline Pipli, N 24 Parganas (SubhRo) & $24.10^{\mathrm{e}} \pm 0.28$ & $0.964^{\mathrm{e}} \pm 0.016$ & $1.205^{c} \pm 0.002$ & $1.687^{b} \pm 0.002$ & $20.241^{b} \pm 0.159$ & $288.8^{\mathrm{e}} \pm 0.12$ \\
\hline Pipli, N 24 Parganas (ShiRo) & $16.36^{\mathrm{bcd}} \pm 0.07$ & $0.818^{\mathrm{cd}} \pm 0.018$ & $0.981^{b} \pm 0.001$ & $1.308^{\mathrm{a}} \pm 0.009$ & $13.251^{\mathrm{a}} \pm 0.009$ & $245.6^{d} \pm 0.09$ \\
\hline Pipli, N 24 Parganas (MadMon) & $29.47^{h} \pm 0.23$ & $1.178^{f} \pm 0.025$ & $2.650^{h} \pm 0.013$ & $3.530^{h} \pm 0.236$ & $22.102^{b c} \pm 0.086$ & $318.1^{9} \pm 0.21$ \\
\hline Pipli, N 24 Parganas (RaKrMo) & $15.59^{\mathrm{ab}} \pm 0.10$ & $0.467^{\mathrm{a}} \pm 0.023$ & $1.241^{c} \pm 0.019$ & $1.870^{b c} \pm 0.055$ & $12.002^{\mathrm{a}} \pm 0.032$ & $203.4^{b} \pm 1.18$ \\
\hline Pipli, N 24 Parganas (DebSar) & $16.05^{\mathrm{abc}} \pm 0.03$ & $0.802^{C} \pm 0.008$ & $0.963^{b} \pm 0.001$ & $1.605^{\mathrm{ab}} \pm 0.003$ & $12.671^{\mathrm{a}} \pm 0.119$ & $227.99^{C} \pm 1.99$ \\
\hline
\end{tabular}

BA/WS, bioavailable arsenic water soluble; BA/WA, bioavailable arsenic weakly adsorbed; BA/SA, bioavailable arsenic specifically adsorbed; $B A / R A$, bioavailable arsenic residual; GW, groundwater

The significant differences among the Mean \pm SE $(n=5)$ values of a column are mentioned by different alphabets (in superscript) $(p<0.05)$

Table 6 Soil physical properties of 15 different sites of three villages in West Bengal

\begin{tabular}{lrrrl}
\hline 15 sites of three villages in the Phase-II & BD $\left(\mathrm{gm} \mathrm{cm}^{-3}\right)$ & \multicolumn{1}{l}{ WHC $(\%)$} & Moisture (\%) & Texture \\
\hline Ghetugachi, Chakdah, Nadia (SusBis) & $1.4^{\mathrm{d}} \pm 0.01$ & $59.01^{\mathrm{e}} \pm 0.02$ & $27.9^{\mathrm{e}} \pm 0.01$ & Clay loam \\
Ghetugachi, Chakdah, Nadia (DipGho) & $1.3^{\mathrm{c}} \pm 0.01$ & $58.08^{\mathrm{d}} \pm 0.02$ & $28.0^{\mathrm{e}} \pm 0.02$ & Clay loam \\
Ghetugachi, Chakdah, Nadia (HarCha) & $1.3^{\mathrm{c}} \pm 0.05$ & $60.02^{\mathrm{h}} \pm 0.08$ & $27.3^{\mathrm{de}} \pm 0.36$ & Clay loam \\
Ghetugachi, Chakdah, Nadia (BikSar) & $1.4^{\mathrm{d}} \pm 0.08$ & $57.94^{\mathrm{cd}} \pm 0.28$ & $27.0^{\mathrm{d}} \pm 0.04$ & Clay loam \\
Ghetugachi, Chakdah, Nadia (PraBis) & $1.4^{\mathrm{d}} \pm 0.01$ & $60.10^{\mathrm{h}} \pm 0.08$ & $27.1^{\mathrm{d}} \pm 0.04$ & Clay loam \\
Gotera, Nadia (SuvMon) & $1.2^{\mathrm{bc}} \pm 0.88$ & $59.82^{\mathrm{g}} \pm 0.01$ & $24.6^{\mathrm{c}} \pm 0.30$ & Clay \\
Gotera, Nadia (ChhMon) & $1.1^{\mathrm{ab}} \pm 0.07$ & $60.21^{\mathrm{h}} \pm 0.06$ & $25.1^{\mathrm{c}} \pm 0.02$ & Clay \\
Gotera, Nadia (AnsMon) & $1.2^{\mathrm{bc}} \pm 0.01$ & $59.33^{\mathrm{f}} \pm 0.08$ & $23.8^{\mathrm{b}} \pm 0.08$ & Clay \\
Gotera, Nadia (PraSan) & $1.2^{\mathrm{bc}} \pm 0.08$ & $59.04^{\mathrm{e}} \pm 0.02$ & $21.9^{\mathrm{a}} \pm 0.06$ & Clay \\
Gotera, Nadia (GanBar) & $1.1^{\mathrm{ab}} \pm 0.05$ & $58.1^{\mathrm{d}} \pm 0.05$ & $25.2^{\mathrm{c}} \pm 0.18$ & Clay \\
Pipli, N 24 Parganas (SubhRo) & $1.3^{\mathrm{c}} \pm 0.07$ & $57.82^{\mathrm{c}} \pm 0.01$ & $32.8^{\mathrm{g}} \pm 0.03$ & Clay \\
Pipli, N 24 Parganas (ShiRo) & $1.3^{\mathrm{c}} \pm 0.72$ & $57.02^{\mathrm{a}} \pm 0.15$ & $33.1^{\mathrm{g}} \pm 0.08$ & Clay \\
Pipli, N 24 Parganas (MadMon) & $1.4^{\mathrm{d}} \pm 0.06$ & $57.63^{\mathrm{b}} \pm 0.03$ & $31.9^{\mathrm{f}} \pm 0.02$ & Clay \\
Pipli, N 24 Parganas (RaKrMo) & $1.2^{\mathrm{bc}} \pm 0.11$ & $57.16^{\mathrm{a}} \pm 0.02$ & $33.7^{\mathrm{h}} \pm 0.05$ & Clay \\
Pipli, N 24 Parganas (DebSar) & $1.3^{\mathrm{c}} \pm 0.05$ & $57.97^{\mathrm{cd}} \pm 0.01$ & $34.0^{\mathrm{h}} \pm 0.01$ & Clay \\
\hline
\end{tabular}

$\mathrm{BD}$, bulk density; WHC, water holding capacity

The significant differences among the Mean \pm SE $(n=5)$ values of a column are mentioned by different alphabets (in superscript) $(p<0.05)$ 


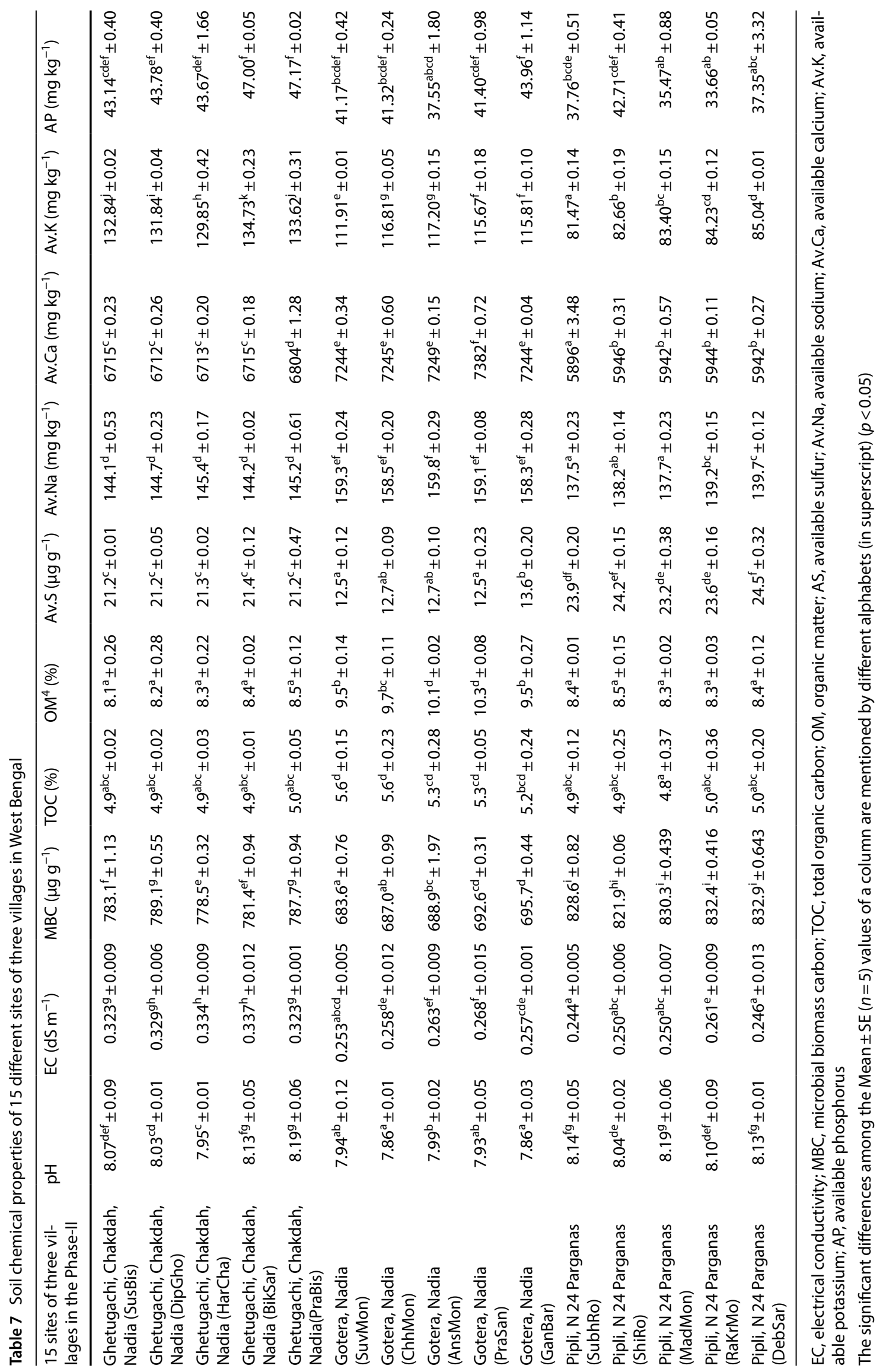


Table 8 Soil enzyme activities of 15 different sites of three villages in West Bengal

\begin{tabular}{|c|c|c|c|c|c|c|c|}
\hline $\begin{array}{l}15 \text { sites of three villages in the } \\
\text { Phase-II }\end{array}$ & $\mathrm{DHA}$ & FDA & BG & APA & Cel & ASA & PA \\
\hline $\begin{array}{l}\text { Ghetugachi,Chakdah, } \\
\text { Nadia (SusBis) }\end{array}$ & $13.01^{\mathrm{cd}} \pm 0.01$ & $14.2^{b} \pm 0.01$ & $22.7^{\mathrm{a}} \pm 0.11$ & $120.13^{9} \pm 0.43$ & $7.51^{\mathrm{ab}} \pm 0.28$ & $60.31^{\mathrm{e}} \pm 0.13$ & $227.77^{\mathrm{ef}} \pm 0.20$ \\
\hline $\begin{array}{l}\text { Ghetugachi,Chakdah, } \\
\text { Nadia (DipGho) }\end{array}$ & $13.16^{b c} \pm 0.05$ & $13.4^{\mathrm{a}} \pm 0.07$ & $22.8^{\mathrm{ab}} \pm 0.14$ & $121.43^{h} \pm 0.14$ & $6.30^{a} \pm 0.24$ & $61.19^{\mathrm{e}} \pm 0.12$ & $226.07^{\mathrm{e}} \pm 0.43$ \\
\hline $\begin{array}{l}\text { Ghetugachi,Chakdah, } \\
\text { Nadia (HarCha) }\end{array}$ & $12.71^{\mathrm{b}} \pm 0.02$ & $13.3^{\mathrm{a}} \pm 0.02$ & $24.8^{\mathrm{b}} \pm 0.18$ & $118.30^{f} \pm 0.11$ & $6.12^{a} \pm 0.72$ & $62.18^{\mathrm{e}} \pm 0.60$ & $229.01^{f} \pm 0.60$ \\
\hline $\begin{array}{l}\text { Ghetugachi,Chakdah, } \\
\text { Nadia (BikSar) }\end{array}$ & $13.71^{d} \pm 0.01$ & $14.9^{c} \pm 0.28$ & $23.1^{\mathrm{ab}} \pm 0.02$ & $119.50^{9} \pm 0.17$ & $8.85^{b c} \pm 0.04$ & $64.80^{f} \pm 0.13$ & $231.89^{f} \pm 0.52$ \\
\hline $\begin{array}{l}\text { Ghetugachi,Chakdah, } \\
\text { Nadia (PraBis) }\end{array}$ & $13.31^{d} \pm 0.01$ & $15.1^{c} \pm 0.05$ & $24.6^{\mathrm{ab}} \pm 0.87$ & $122.07^{h} \pm 0.02$ & $9.15^{\mathrm{bcd}} \pm 0.07$ & $65.67^{\mathrm{fg}} \pm 0.11$ & $231.72^{f} \pm 0.96$ \\
\hline $\begin{array}{l}\text { Gotera, Nadia } \\
\text { (SuvMon) }\end{array}$ & $18.10^{e} \pm 0.05$ & $19.5^{f} \pm 0.20$ & $31.7^{d} \pm 0.28$ & $19.09^{\mathrm{a}} \pm 0.15$ & $9.30^{\text {cd }} \pm 0.65$ & $43.73^{a} \pm 0.13$ & $104.78^{\mathrm{a}} \pm 0.23$ \\
\hline $\begin{array}{l}\text { Gotera, Nadia } \\
\text { (ChhMon) }\end{array}$ & $18.25^{f} \pm 0.02$ & $20.6^{\mathrm{gh}} \pm 0.17$ & $32.4^{\mathrm{de}} \pm 0.27$ & $20.43^{b} \pm 0.17$ & $10.22^{\text {cde }} \pm 0.17$ & $45.38^{\mathrm{ab}} \pm 0.31$ & $104.68^{\mathrm{a}} \pm 0.06$ \\
\hline $\begin{array}{l}\text { Gotera, Nadia } \\
\text { (AnsMon) }\end{array}$ & $18.23^{\mathrm{a}} \pm 0.06$ & $21.0^{\mathrm{h}} \pm 0.04$ & $33.7^{\mathrm{ef}} \pm 0.11$ & $21.70^{C} \pm 0.21$ & $11.32^{\mathrm{ef}} \pm 0.31$ & $46.77^{b c} \pm 0.12$ & $106.12^{\mathrm{a}} \pm 0.42$ \\
\hline $\begin{array}{l}\text { Gotera, Nadia } \\
\text { (PraSan) }\end{array}$ & $18.34 f^{9} \pm 0.03$ & $18.0^{d} \pm 0.02$ & $32.0^{\mathrm{de}} \pm 0.57$ & $19.84^{\mathrm{ab}} \pm 0.02$ & $10.78^{\mathrm{de}} \pm 0.20$ & $48.83^{\mathrm{cd}} \pm 0.06$ & $109.65^{b} \pm 0.06$ \\
\hline $\begin{array}{l}\text { Gotera, Nadia } \\
\text { (GanBar) }\end{array}$ & $18.38^{\mathrm{g}} \pm 0.12$ & $19.4^{f} \pm 0.37$ & $29.4^{c} \pm 0.43$ & $20.32^{\mathrm{b}} \pm 0.29$ & $10.04^{\text {cde }} \pm 0.36$ & $49.34^{d} \pm 0.16$ & $110.23^{b} \pm 0.06$ \\
\hline Pipli, N 24 Parganas (SubhRo) & $20.08^{\mathrm{h}} \pm 0.15$ & $19.5^{f} \pm 0.01$ & $33.0^{\text {def }} \pm 0.03$ & $23.17^{\mathrm{de}} \pm 0.17$ & $12.87^{\mathrm{fg}} \pm 0.11$ & $62.08^{\mathrm{e}} \pm 0.44$ & $176.07^{c} \pm 0.03$ \\
\hline $\begin{array}{l}\text { Pipli, N } 24 \text { Parganas } \\
\text { (ShiRo) }\end{array}$ & $20.25^{f} \pm 0.23$ & $20.2^{g} \pm 0.03$ & $33.2^{\text {def }} \pm 0.28$ & $19.53^{\mathrm{ab}} \pm 0.31$ & $13.43^{9} \pm 0.04$ & $64.78^{f} \pm 1.35$ & $179.44^{\mathrm{cd}} \pm 0.01$ \\
\hline Pipli, N 24 Parganas (MadMon) & $20.35^{i} \pm 0.03$ & $21.0^{\mathrm{h}} \pm 0.01$ & $34.1^{f} \pm 0.24$ & $23.53^{\mathrm{de}} \pm 0.31$ & $13.78^{9} \pm 0.17$ & $67.68^{g h} \pm 0.23$ & $179.54^{d} \pm 0.01$ \\
\hline Pipli, N 24 Parganas (RaKrMo) & $20.41^{i} \pm 0.06$ & $21.8^{i} \pm 0.01$ & $34.2^{f} \pm 0.63$ & $22.77^{\mathrm{cd}} \pm 0.14$ & $9.85^{\mathrm{cd}} \pm 0.16$ & $69.04^{h} \pm 0.58$ & $180.20^{d} \pm 0.04$ \\
\hline Pipli, N 24 Parganas (DebSar) & $20.08^{h} \pm 0.05$ & $18.7^{\mathrm{e}} \pm 0.14$ & $33.3^{\mathrm{def}} \pm 0.36$ & $23.97^{\mathrm{e}} \pm 0.05$ & $10.13^{\text {cde }} \pm 0.38$ & $69.72^{h} \pm 0.18$ & $174.90^{c} \pm 0.05$ \\
\hline
\end{tabular}

DHA (Dehydrogenase activity) as $\mu \mathrm{g}$ TPF g soil ${ }^{-1} 24 \mathrm{~h}^{-1}$; FDA (fluorescein diacetate) as $\mu \mathrm{g}$ Fluorescein $\mathrm{g}$ soil ${ }^{-1} 2 \mathrm{~h}^{-1}$; APA (alkaline phosphatase activity) as $\mu \mathrm{g} \rho$-NP g soil ${ }^{-1} \mathrm{~h}^{-1}$; BG ( $\beta$-glucosidase) as $\mu \mathrm{g} \rho$-NP g soil ${ }^{-1} \mathrm{~h}^{-1}$; PA (protease activity) as $\mu \mathrm{g} \mathrm{Tyr} \mathrm{g} \mathrm{soil}{ }^{-1} 2 \mathrm{~h}^{-1}$; CeA (cellulase activity) as $\mu \mathrm{g}$ glucose $\mathrm{g}$ soil ${ }^{-1} 24 \mathrm{~h}^{-1} ;{ }^{7} \mathrm{ASA}$ (arylsulfatase) as $\mu \mathrm{g} \rho-\mathrm{NP} \mathrm{g} \mathrm{soil}{ }^{-1} \mathrm{~h}^{-1}$

The significant differences among the Mean \pm SE $(n=5)$ values of a column are mentioned by different alphabets (in superscript) $(p<0.05)$

Fig. 2 PCA biplot of soil arsenic content of ten villages in West Bengal. BA, bioavailable; WA, weakly adsorbed; SA, specifically adsorbed; WS, water soluble; and RA, residual
Biplot (axes F1 and F2: $98.74 \%$ )

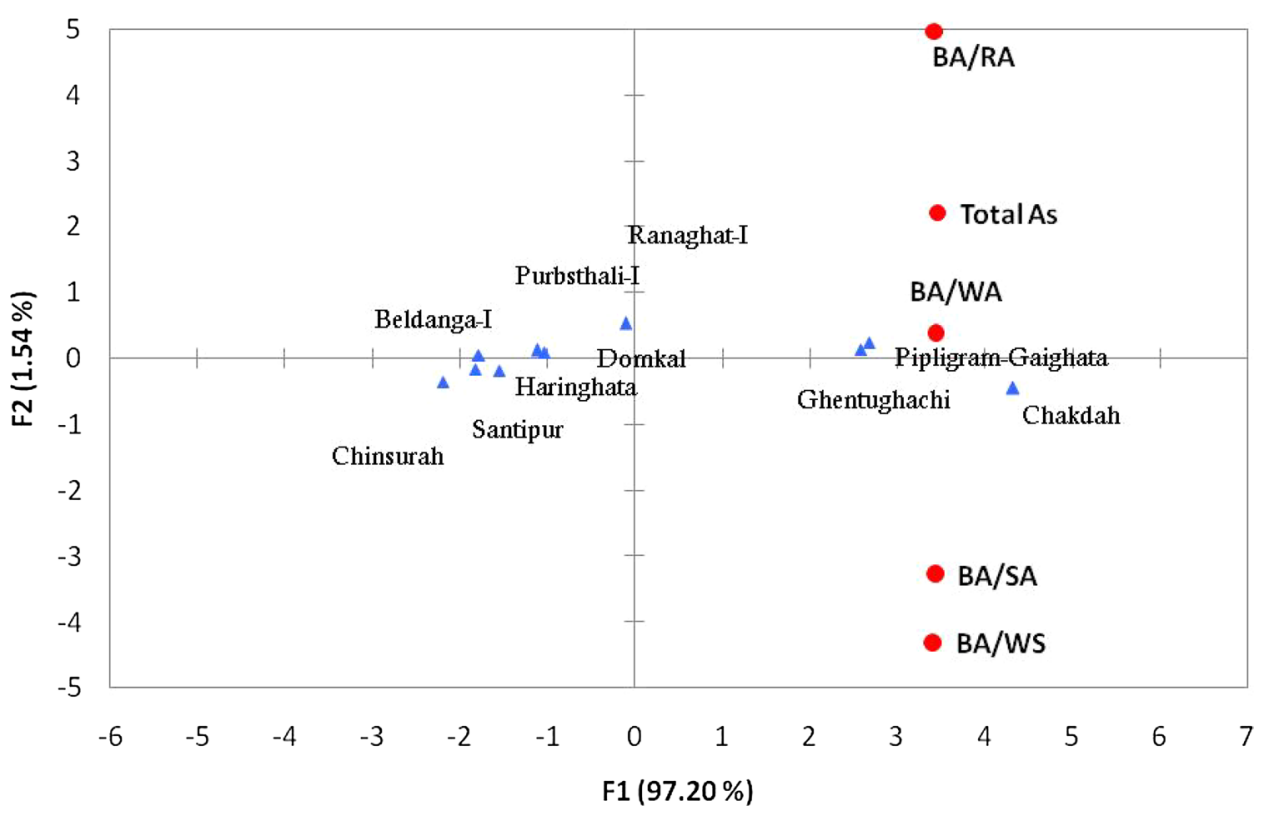


Fig. 3 PCA biplot of soil arsenic and physical properties of ten villages in West Bengal. WHC, water holding capacity; BD and bulk density; moisture

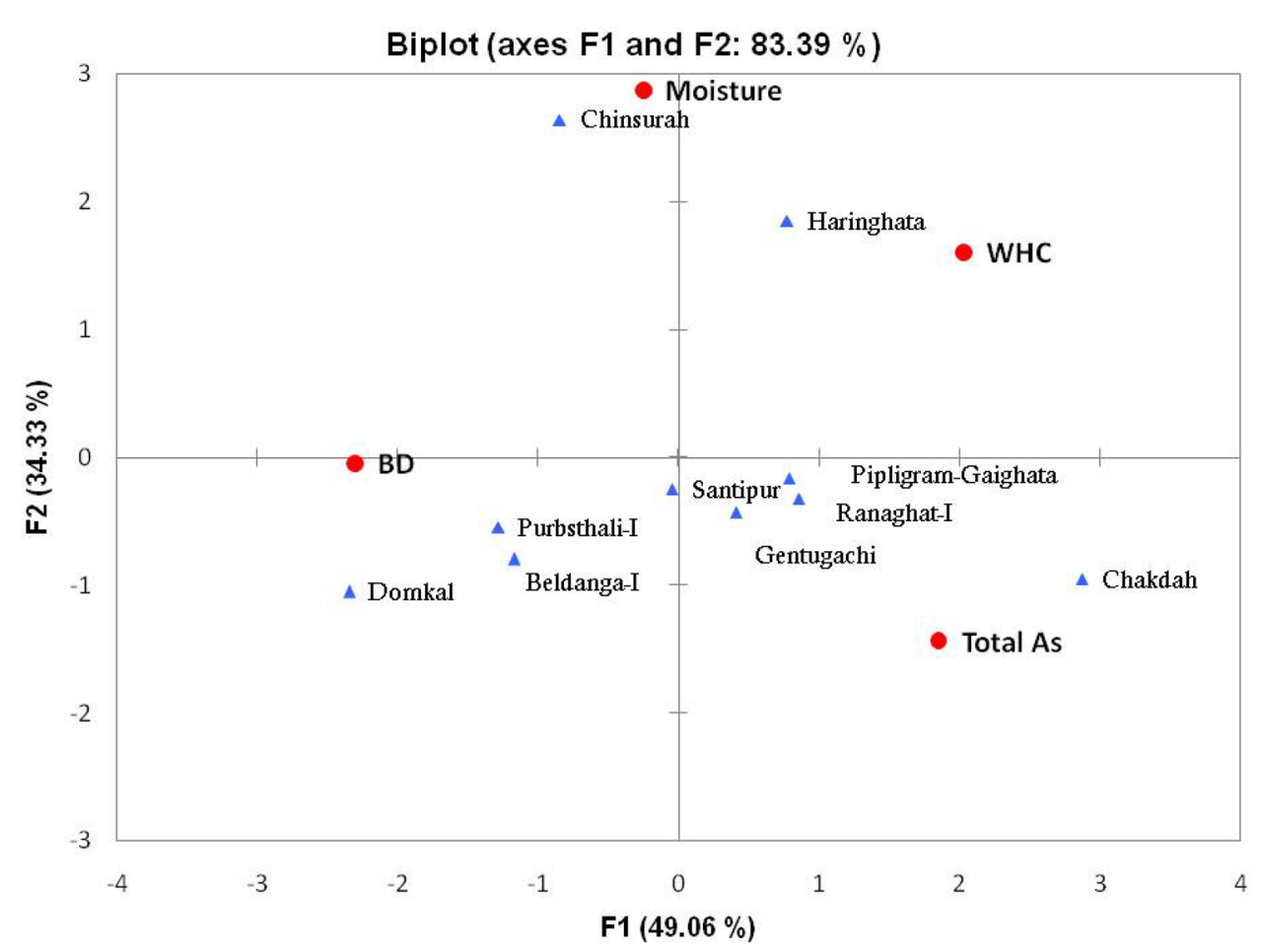

Fig. 4 PCA biplot of soil arsenic and chemical properties of ten villages in West Bengal. MBC, microbial biomass carbon; Av-Na, available sodium; Av-Ca, available calcium; Av-K, available potassium; TOC, total organic carbon; EC, electrical conductivity; $A P$, available phosphorus; AS, available sulfate-sulfur; and OM, organic matter

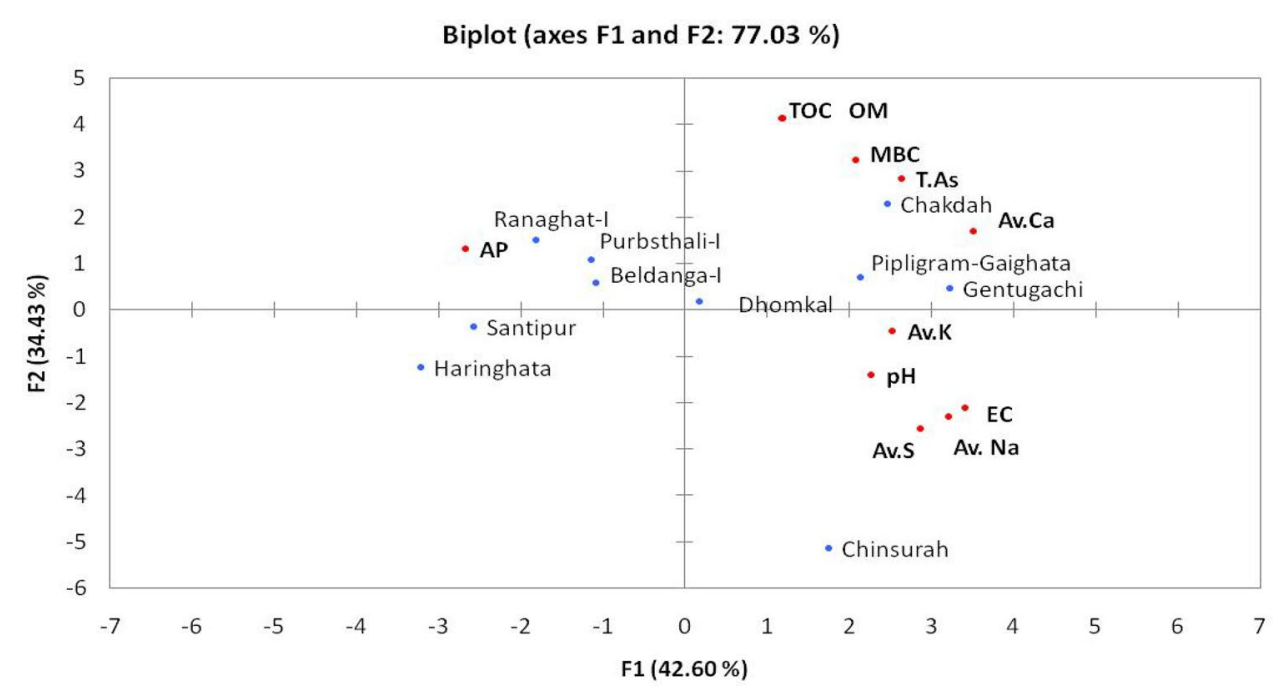

content and different soil physicochemical properties and enzyme activities.

\section{Discussion}

Out of the total ten villages surveyed in five different districts of West Bengal during the Phase-l, three villages (Ghentughachi, Gotera and Pipligram) exhibited higher arsenic contamination (ranged $24.7-31.1 \mathrm{mg} \mathrm{kg}^{-1}$ ) in the paddy soils above the threshold limit $\left(20 \mathrm{mg} \mathrm{kg}^{-1}\right)$ given by FAO. The arsenic content in groundwater ranged between 112.3 and $558.02 \mu \mathrm{g} \mathrm{I}^{-1}$, which also exhibited higher arsenic contamination in groundwater above the threshold limit of $50 \mathrm{\mu g} \mathrm{I}^{-1}$ given by the Indian Standards for Drinking Water, second revision of IS-10500, 2004. Several studies specify that irrigation with As-laden groundwater is building up the soil As levels $[17,31]$. The study showed arsenic water soluble, weakly adsorbed fraction, specifically sorbed, and the residual fraction in the range of $5-7 \%, 7-12 \%, 8-15 \%$, and $75-85 \%$, respectively, in the soil samples. The first fraction represents water-soluble arsenic, which is the most mobile form of arsenic. The second fraction showed weakly exchangeable arsenic, 
Fig. 5 PCA biplot of soil arsenic and enzyme activities of ten villages in West Bengal. FDA, fluorescein diacetate; APA alkaline phosphatase; $\mathrm{PA}$, protease; $\mathrm{CeA}$, cellulase; $\mathrm{BG}$, $\beta$-glucosidase; DHA, dehydrogenase; and ASA, arylsulfatase
Fig. 6 PCA biplot of soil and groundwater arsenic content of 15 different sites of three villages in West Bengal. BA, bioavailable; WA, weakly adsorbed; SA, specifically adsorbed; WS, water soluble; $\mathrm{RA}$, residual; and total As (GW), total arsenic groundwater
Biplot (axes F1 and F2: $76.14 \%$ )

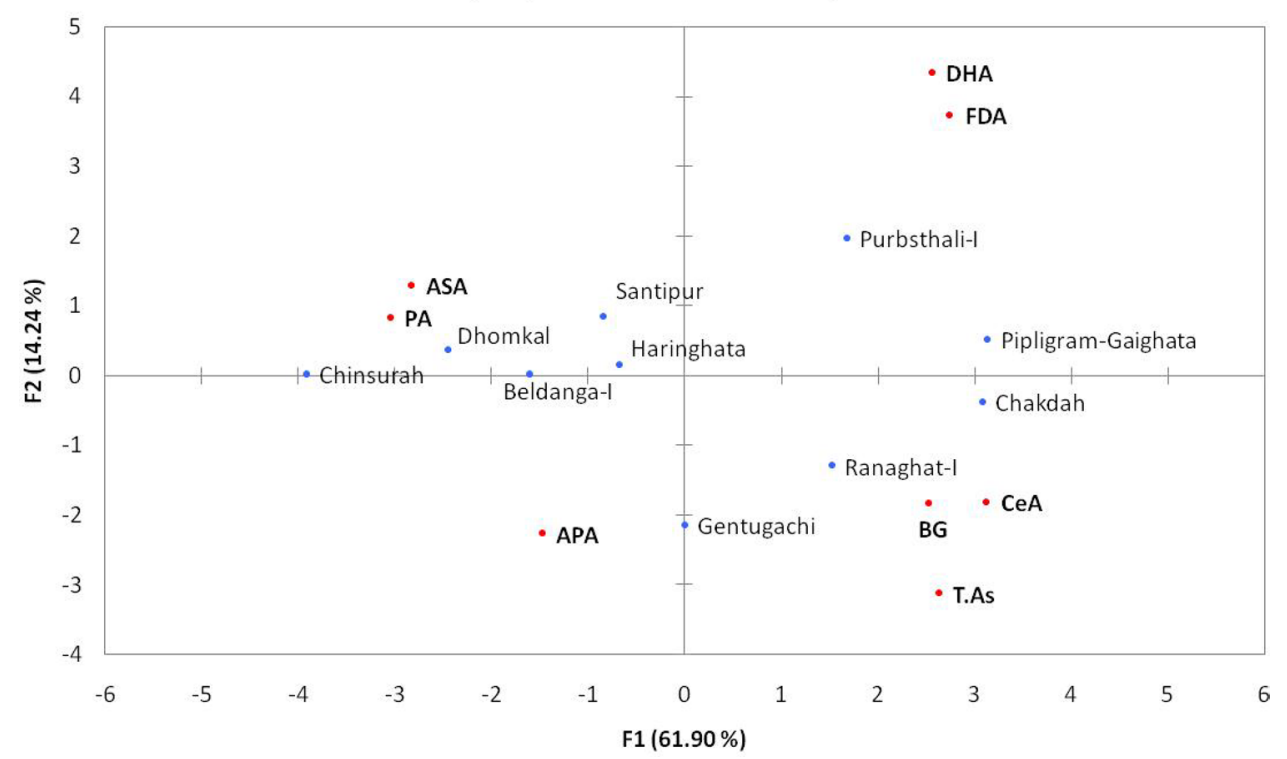

Biplot (axes F1 and F2: $85.73 \%$ )

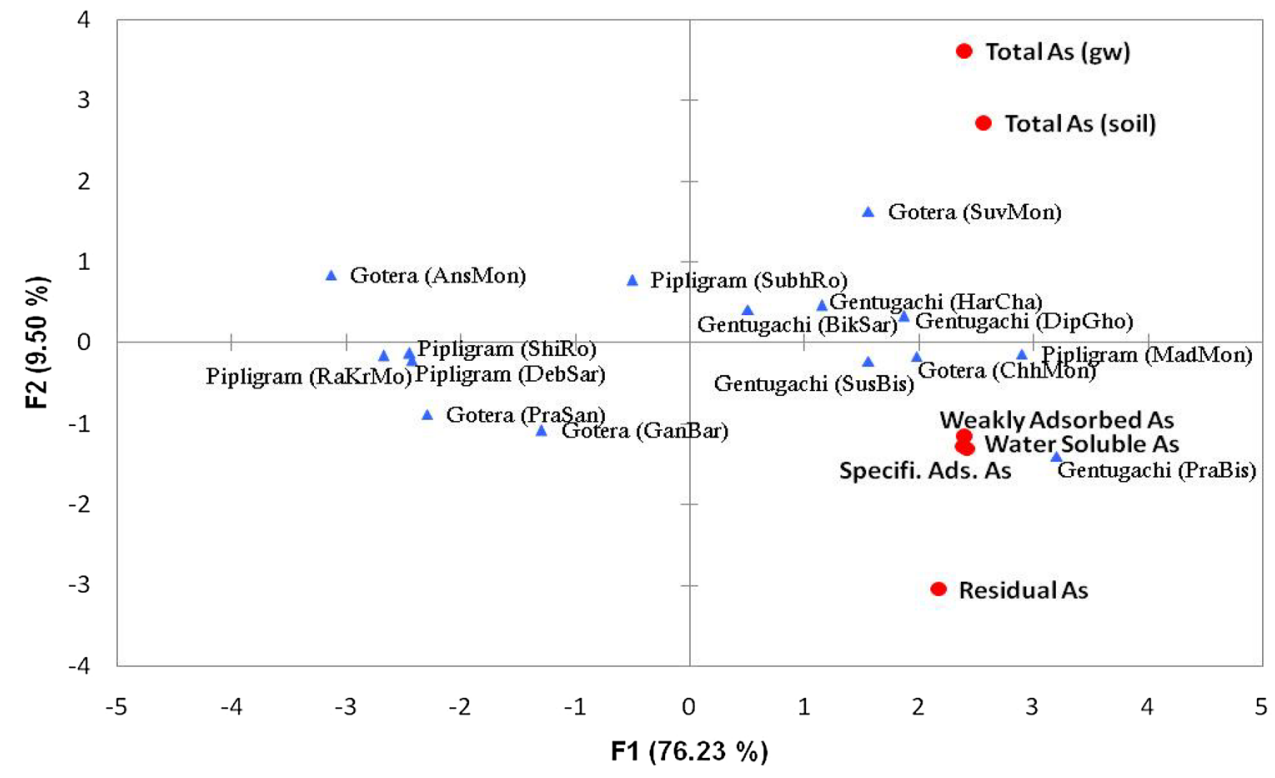

which binds to soil forming outer sphere complexes. This fraction can be released easily into soil water through ion exchange processes [44]. The specifically sorbed fraction ranged from 8 to $15 \%$, which represents the strongly bound inner-sphere surface complex between arsenic and metal oxide, which might be extracted into soil pore water by competitive ligand exchange [42]; Yolcubl et al. [45]. The soil arsenic contents differed largely depending on the underlying site-specific soil properties. Soil properties have a significant influence on the speciation, bioavailability, and solubility of As (Bissen and Frimmel et al. [5, 19]. In the study, key soil parameters that have been influencing the bioavailability of soil arsenic were $\mathrm{pH}$, phosphorus, sulfur, and organic carbon content. Arsenic mobility and bioavailability may vary within the $\mathrm{pH}$ range between 6.5 and 8.5 depending upon the oxidizing as well as reducing conditions in soil (Dzombak et al. [14].

The predominance of As fractions is observed in an order of residual > specifically sorbed > weakly sorbed/ exchangeable $>$ water soluble. The residual fraction of soil arsenic is based upon co-precipitation, adsorption, surface complex formation, ion exchange, and chemical bonding. Although As associated with Fe and Mn (hydro) oxides presents low mobility in a non-acidic oxidizing 
Fig. 7 PCA biplot of soil arsenic and physical properties of 15 different sites of three villages in West Bengal. WHC, water holding capacity; $B D$, bulk density; moisture; and total As (GW), total arsenic groundwater

\section{Biplot (axes F1 and F2: $79.85 \%$ )}

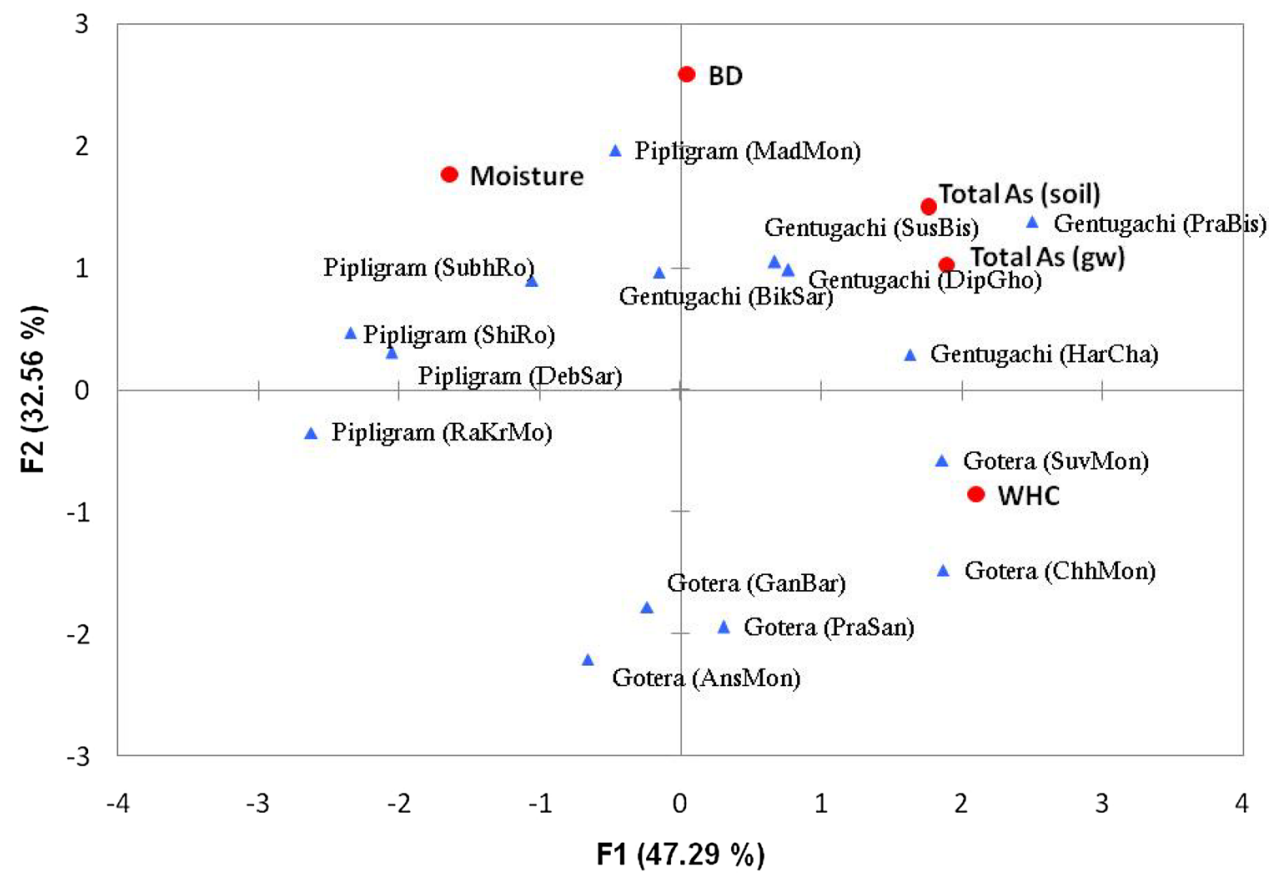

Fig 8 PCA biplot of soil arsenic and chemical properties of 15 different sites of three villages in West Bengal. MBC, microbial biomass carbon; $\mathrm{Av}-\mathrm{Na}$, available sodium; Av-Ca, available calcium; Av-K, available potassium; TOC, total organic carbon; EC, electrical conductivity; AP, available phosphorus; AS, available sulfate-sulfur; OM, organic matter; and total As (GW), total arsenic groundwater
Biplot (axes F1 and F2: $86.46 \%$ )

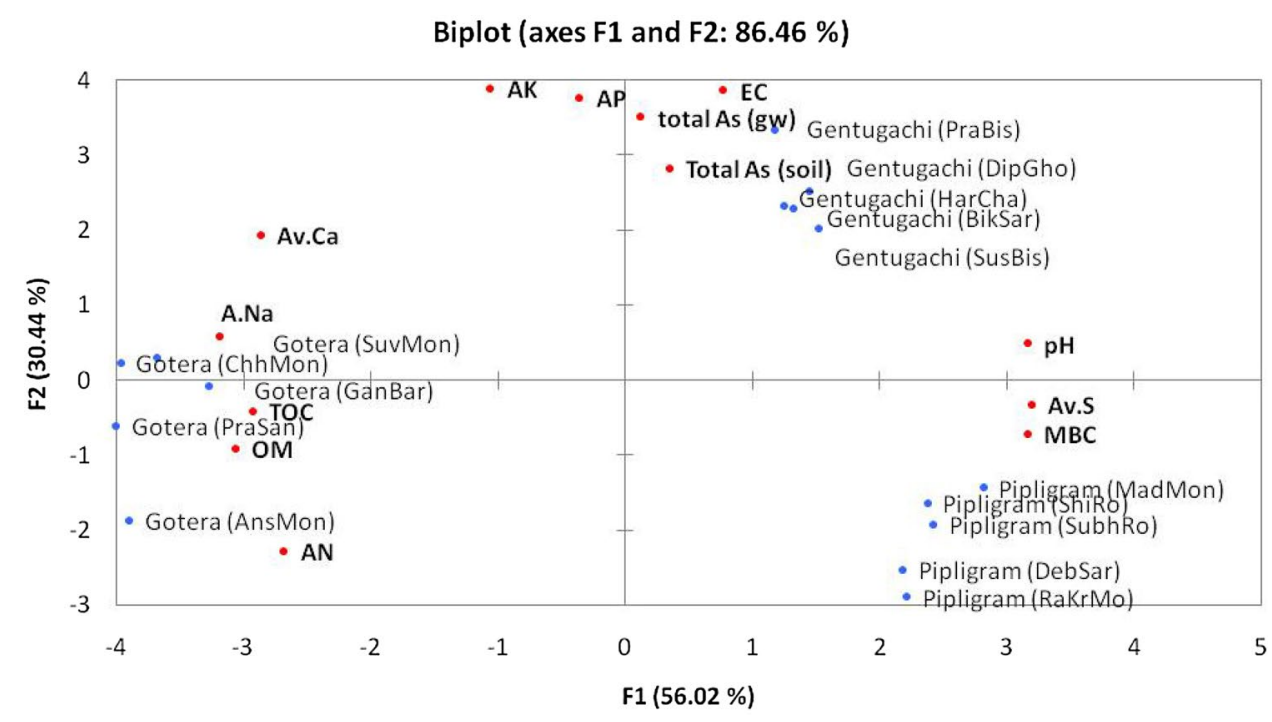

environments, there is a risk of its release, when soil redox potential or $\mathrm{pH}$ changes. Changes in the ionic composition or influencing adsorption-desorption reactions could cause mobilization of As from this fraction. Significant correlations were obtained between the total soil As content and its specifically sorbed, weakly sorbed or exchangeable, water soluble, and residual fractions. Total content of arsenic present in soils cannot provide a precise index for evaluating their toxicity to soil and crop cultivation in the contaminated soils. The water-soluble As was considered to be the most labile fraction and important for toxicity concern. The major amount of the As fraction holds up strongly or present in immobile residual forms binding to high clay matrices in the soil. Soil As content and its toxicity depend upon its various forms from easily leachable to recalcitrant ones due to its interactions with different soil components.

The TOC and OM content of soil found positively and significantly correlated with the bioavailability of arsenic in the soil. The correlation between As adsorption by humic acid is well known, although the accurate retention mechanisms remain contentious [21], and some experiments indicate that the occurrence of organic matter causes the reverse effect, competing with As for adsorption sites on 
Fig. 9 PCA biplot of soil arsenic and enzyme activities of 15 different sites of three villages in West Bengal. FDA, fluorescein diacetate hydrolase; APA, alkaline phosphatase; $\mathrm{PA}$, protease; $\mathrm{CeA}$, cellulase; $\mathrm{BG}$, $\beta$-glucosidase; DHA, dehydrogenase; ASA, arylsulfatase; and total As (GW), total arsenic groundwater
Biplot (axes F1 and F2: $82.66 \%$ )

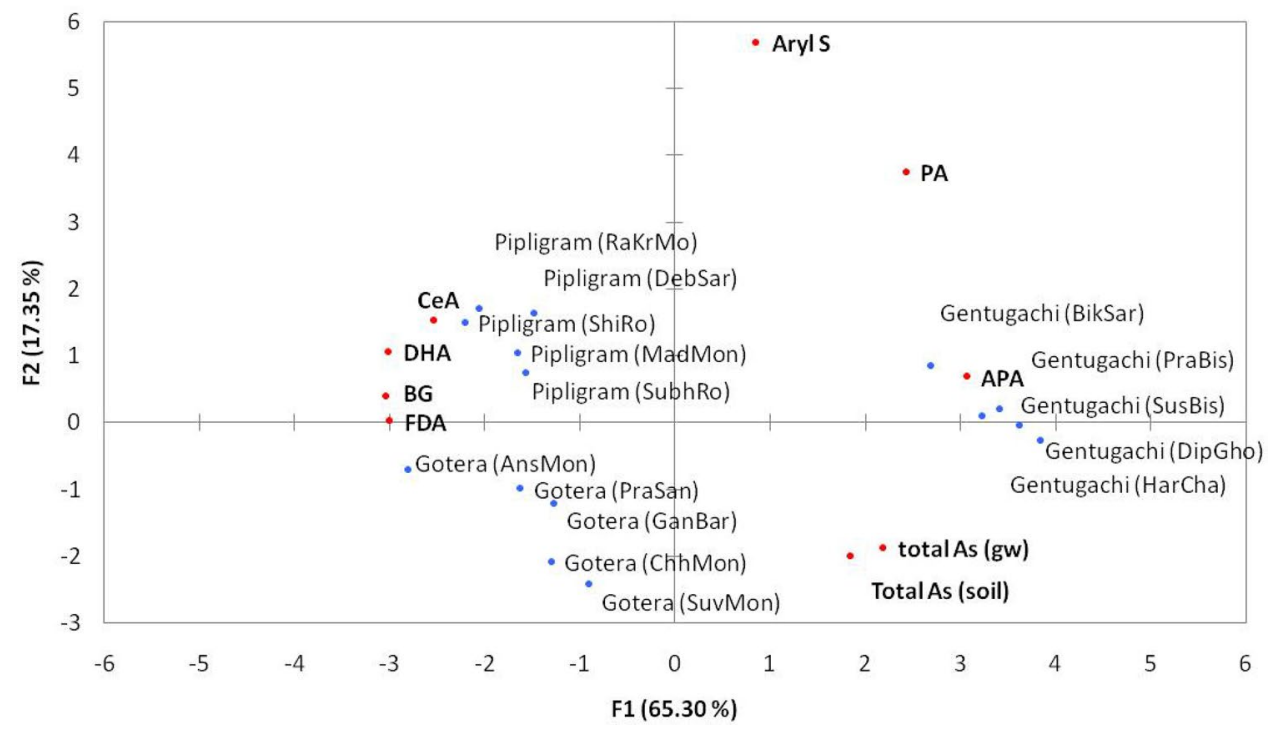

iron oxide surfaces [30]. An increase in OM can augment desorption of As from solid phase [30,41], because it can enhance microbial activity and decrease the soil redox potential, which favors the reductive dissolution of Fe-oxyhydroxide linked to OM. Additionally, dissolved OM leads to an augment As mobility, as it contains negative charges, which have a high potential to contend with As for sorption sites in soil [22]. Higher arsenic-contaminated soil samples were rich in organic matter. Increases in organic matter may enhance the desorption of As from the solid phase by increasing microbial activity and reductive dissolution of Fe-oxyhydroxide. This process may lead to the sorption of humic anions on Fe oxides resulting in competition for sorption sites and preventing As sorption, or inducing As desorption and thus increase the mobility of As in the soil [33].

It is also found that higher calcium content may be attributed to the formation of stable Ca-arsenate precipitate [29]. The increase in soil arsenic bioavailability was correlated with a decrease in the concentration of available phosphorus content in soil, because it competes with arsenic for sorption sites in soils [35]. In Ghentughachi, Gotera, and Pipligram sites, a negative correlation was found between $P$ and As because of the P-induced As mobilization and bioavailability by competitive displacement of $\mathrm{AsO}_{4}$ by $\mathrm{PO}_{4}$ at sorption sites [36]. The negative correlation found between $\mathrm{S}$ and high As content in soil among all the study sites. $S$ and As share similar chemical and biological redox transformation, and their biogeochemical cycles are often interconnected [18]. Arsenic content and its build up in soils significantly hampered the soil enzyme activities. It has been reported that arsenite inactivates enzymes through reacting with $-\mathrm{SH}$ groups and formed As sulfide [11, 36]. Soil enzyme activity is a sensitive indicator of soil quality. The BG activity is reported to be significantly correlated with SOC (Bandick et al. [2] of the study. The results also showed that DHA, FDA, CeA, and BG activities were increased in the highest arsenic-contaminated sites, which may be due to high $\mathrm{OM}$ content and corresponding increase in soil MBC content. This needs further investigation.

\section{Conclusion}

The study revealed that soil arsenic contents and its bioavailable fractions found strongly correlated with different enzymatic activities and physicochemical properties of soil. Soil As contamination in Gotera, Pipligram, and Ghentughachi observed as most alarming due to highest bioavailable As fractions reported compared to remaining other studied sites in West Bengal. Bioavailable soil arsenic content was in order of water soluble < weakly adsorbed $<$ specifically adsorbed. The PCA results pointed out that $\mathrm{OM}, \mathrm{AP}, \mathrm{AS}, \mathrm{Ca}$, and $\mathrm{pH}$ are the most correlated soil properties to the different bioavailable soil As contents, while BD and AP negatively correlated and $\mathrm{OM}$ and $\mathrm{Ca}$ positively correlated with As content in soil controlling levels of bioavailable As contents. Further, arsenic speciation can be studied vis-à-vis bioavailability of arsenic in soils, which may confer more about toxicity of different bioavailable fractions in soil. These results may help to frame soil-based remediation strategies to reduce arsenic availability in contaminated soil. 
Acknowledgements Authors are grateful to Director CSIR-NBRI for the support. Suman B Singh is thankful to the University Grants Commission (UGC), Govt. of India, for the fellowship provided (23/12/2012 (ii) EU-V) and also to the Academy of Scientific and Innovative Research (AcSIR) from where she is pursuing her doctorate. The authors are thankful to the Department of Biotechnology (DBT) for the financial grant (BT/PR-12764/BCE/8/1119/2015) to carry out the study.

Author contributions Both the authors have made a joint contribution in all aspects to carry out and complete the study.

Funding Department of Biotechnology (DBT), Government of India (BT/PR-12764/BCE/8/1119/2015)

\section{Compliance with ethical standard}

Conflict of interest The authors have no competing interests to declare.

\section{References}

1. Ahamed $S$, Kumar Sengupta $M$, Mukherjee $A$, Amir Hossain $M$, Das B, Nayak B, Pal A, Chandra Mukherjee S, Pati S, Nath Dutta R, Chatterjee G, Mukherjee A, Srivastava R, Chakraborti D (2006) Arsenic groundwater contamination and its health effects in the state of Uttar Pradesh (UP) in upper and middle Ganga plain. India: a severe danger. Sci Total Environ 370:310-322

2. Bandick AK, Dick RP (1999) Field management effects on soil enzyme activities. Soil Biol Biochem 31:1471-1479

3. Bhattacharya P, Pal R (2011) Response of cyanobacteria to arsenic toxicity. J Appl Phycol 23:293-299

4. Bhattacharya P, Welch AH, Stollenwerk KG, McLaughlin MJ, Bundschuh J, Panaullah G (2007) Arsenic in the environment: biology and Chemistry. Sci Total Environ 379:109-120

5. Bissen M, Frimmel FH (2003) Arsenic - a review. Part I: occurrence, toxicity, speciation, mobility. Acta Hydroch Hydrob 31:9-18

6. Black CA (1965) Method of Soil Analysis Part 2 Chemical and Microbiological Properties. American Society of Agronomy. Inc Publisher, Madison

7. Bundschuh J, Nath B, Bhattacharya P, Liu CW, Armienta MA, Moreno López MV, Lopez DL, Jean JS, Cornejo L, Lauer Macedo LF, Filho AT (2012) Arsenic in the human food chain: the Latin American perspective. Sci Total Environ 429:92-106

8. Casentini B, Hug SJ, Nikolaidis NP (2011) Arsenic accumulation in irrigated agricultural soils in Northern Greece. Sci Total Environ 409:4802-4810

9. Chakraborti D, Singh SK, Rahman MM, Dutta RN, Mukherjee SC, Pati S, Kar PB (2018) Groundwater arsenic contamination in the Ganga River Basin: a future health danger. Int J Environ Res Public Health 15:180. https://doi.org/10.3390/ijerph15020180

10. Chauhan VS, Yunus M, Sankararamakrishnan N (2012) Geochemistry and mobilization of arsenic in Shuklaganj area of KanpurUnnao district, Uttar Pradesh, India. Environ Monit Assess 184:4889-4901

11. Das S, Jean SH, Kar S, Chakraborty S (2013) Effect of arsenic contamination on bacterial and fungal biomass and enzyme activities in tropical arsenic-contaminated soils. Biol Fertil Soils 49:757-765

12. Datta BK, Mishra A, Singh A, Sar TK, Sarkar S, Bhatacharya A, Chakraborty AK, Mandal TK (2010) Chronic arsenicosis in cattle with special reference to its metabolism in arsenic endemic village of Nadia district West Bengal India. Sci Total Environ 409:284-288

13. Dick RP (2011) Methods of soil enzymology. Issue 9 of soil science society of America book series. Soil Science Society of America. Inc Publisher, Madison

14. Dzombak DD, Morel FMM (1990) Surface complex modeling: Hydrous Ferric Oxide. Wiley, New York

15. FAO (2006) Arsenic contamination of irrigation water, soil and crops in Bangladesh: Risk implications for sustainable agriculture and food safety in Asia. In: Heikens A (ed) Regional office for Asia and The Pacific, Rap publication 2006/20. Food and Agriculture Organization of the United Nation (FAO), Bangkok, Thailand

16. Goh KH, Lim TT (2005) Arsenic fractionation in a fine soil fraction and influence of various anions on its mobility in the subsurface environment. Appl Geochem 20:229-239

17. Hossain MB, Jahiruddin M, Panaullah GM, Loeppert RH, Islam MR, Duxbury JM (2008) Spatial variability of arsenic concentration in soils and plants, and its relationship with iron, manganese and phosphorus. Environ Pollut 156:739-744

18. Hu ZY, Zhu YG, Li M, Zhang LG, Cao ZH, Smith FA (2007) Sulfur (S)-induced enhancement of iron plaque formation in the rhizosphere reduces arsenic accumulation in rice (Oryza sativa L.) seedlings. Environ Pollut 147:387-393

19. Juhasz AL, Naidu R, Zhu YG, Wang LS, Jiang JY, Cao ZH (2003) Toxicity issues associated with geogenic arsenic in the groundwater-soil-plant-human continuum. B Environ Contam Tox 71:1100-1107

20. Khan S, Rauf R, Muhammad S, Qasim M, Din I (2016) Arsenic and heavy metals health risk assessment through drinking water consumption in the Peshawar District, Pakistan. Hum Ecol Risk Assess 22:581-596

21. Kumpiene J, Lagerkvist A, Maurice $C$ (2008) Stabilization of As, $\mathrm{Cr}, \mathrm{Cu}, \mathrm{Pb}$ and $\mathrm{Zn}$ in soil using amendments-a review. Waste Manage 28:215-225

22. Lin CF, Wu CH, Lai HT (2008) Dissolved organic matter and arsenic removal with coupled chitosan/UF operation. Sep Purif Technol 60:292-298

23. McLaren RG, Naidu R, Smith J, Tiller KG (1998) Fractionation and distribution of arsenic in soils contaminated by cattle dip. J Environ Qual 27:348-354

24. Murcott S (2012) Arsenic contamination in the world. IWA publishing

25. Naujokas MF, Anderson B, Ahsan H, Aposhian HV, Graziano JH, Thompson C, Suk WA (2013) The broad scope of health effects from chronic arsenic exposure: update on a worldwide public health problem. Environ Health Perspect 121:295-302

26. Olsen SR, Cole CV, Watanabe FS, Dean LA (1954) Estimation of available phosphorus in soils by extraction with sodium bicarbonate, vol 19. Circular, Washington, p 19 (US Department of Agriculture)

27. Pribyl DW (2010) A critical review of the conventional SOC to SOM conversion factor. Geoderma 156:75-83

28. Punshon T, Jackson BP, Meharg AA, Warczack T, Scheckel K, Guerinot ML (2017) Understanding arsenic dynamics in agronomic systems to predict and prevent uptake by crop plants. Sci Total Environ 581-582:209-220

29. Rafiq M, Shahid M, Abbas G, Shamshad S, Khalid S, Niazi NK, Dumat C (2017) Comparative effect of calcium and EDTA on arsenic uptake and physiological attributes of Pisum sativum. Int J Phytoremediat 19:662-669

30. Redman AD, Macalady DL, Ahmann D (2002) Natural organic matter affects arsenic speciation and sorption onto hematite. Environ Sci Technol 36:2889-2896

31. Roberts LC, Hug SJ, Dittmar J, Voegelin A, Saha GC, Ali MA, Badruzzaman AB, Kretzschmar R (2007) Spatial distribution and 
temporal variability of arsenic in irrigated rice fields in Bangladesh. 1. Irrigation water. Environ Sci Technol 41:5960-5966

32. Sadiq M (1997) Arsenic chemistry in soils: an overview of thermodynamic predictions and field observations. Water Air Soil Pollut 93:117-136

33. Sahoo PK, Kim K (2013) A review of the arsenic concentration in paddy rice from the perspective of geoscience. Geosci J 17:107-122

34. Sinha B, Bhattacharyya K (2011) Retention and release isotherm of arsenic in arsenic-humic/fulvic equilibrium study. Biol Fertil Soils 47:815-822

35. Smith E, Naidu R, Alston AM (2002) Chemistry of inorganic arsenic in soils. J Environ Qual 31:557-563

36. Smith SE, Christophersen HM, Pope S, Smith FA (2010) Arsenic uptake and toxicity in plants: integrating mycorrhizal influences. Plant Soil 327:1-21

37. Srivastava PK, Gupta M, Upadhyay RK, Sharma S, Shikha Singh N, Tewari SK, Singh B (2012) Effects of combined application of vermicompost and mineral fertilizer on the growth of Allium cepa L. and soil fertility. J Plant Nutr Soil Sc 175:101-107

38. Srivastava PK, Singh PC, Gupta M, Sinha A, Vaish A, Shukla A, Singh N, Tewari SK (2011) Influence of earthworm culture on fertilization potential and biological activities of vermicomposts prepared from different plant wastes. J Plant Nutr Soil Sci 174:420-429

39. Srivastava PK, Vaish A, Dwivedi S, Chakrabarty D, Singh N, Tripathi RD (2011) Biological removal of arsenic pollution by soil fungi. Sci Total Environ 409:2430-2442
40. Tabatabai MA, Bremner JM (1972) Distribution of total and available sulfur in selected soils and soil profiles. Agron J 64:40-44

41. Turpeinen R, Pantsar-Kallio M, Häggblom M, Kairesalo T (1999) Influence of microbes on the mobilization, toxicity and biomethylation of arsenic in soil. Sci Total Environ 236:173-180

42. Wenzel WW, Kirchbaumer N, Prohaska T, Stingeder G, Lombi E, Adriano DC (2001) Arsenic fractionation in soils using an improved sequential extraction procedure. Anal Chim Acta 436:309-323

43. WHO (2005) Water sanitation and health. Guidelines for drinking water quality. 3rd edn. World Health Organization

44. Yan XL, Lin LY, Liao XY, Zhang WB, Wen Y (2013) Arsenic stabilization by zero-valent iron, bauxite residue, and zeolite at a contaminated site planting Panax notoginseng. Chemosphere 93:661-667

45. Yolcubal I, Akyol NH (2008) Adsorption and transport of arsenate in carbonate-rich soils: coupled effects of nonlinear and ratelimited sorption. Chemosphere 73:1300-1307

Publisher's Note Springer Nature remains neutral with regard to jurisdictional claims in published maps and institutional affiliations. 